QUARTERLY OF APPLIED MATHEMATICS

VOLUME LXIX, NUMBER 4

DECEMBER 2011, PAGES 651-676

S 0033-569X(2011)01225-7

Article electronically published on June 29, 2011

\title{
ELECTRODYNAMICAL INSTABILITY OF LAYERED STRUCTURES
}

\author{
BY \\ A. M. BLOKHIN (Institute of Mathematics, Novosibirsk State University, Novosibirsk, 630090, \\ Russia) \\ AND \\ R. E. SEMENKO (Novosibirsk State University, Novosibirsk, 630090, Russia)
}

\begin{abstract}
We are concerned with a hydrodynamical model of layered structures at the presence of an electric current. We formulate a linearized stability problem for layered structures and prove that the solutions of this problem grow infinitely, which means the destruction of layered structures at the presence of the small-amplitude alternating electric current.
\end{abstract}

1. Introduction. It is known that the increase of oil reservoir performance is of great importance for modern power engineering. The difficulty in the resolution of this problem is that, in chinked zones of exploiting collectors, there appear water-oil layered structures which block transport structures of collectors and, therefore, eject significant petroferous areas from modes of water replacement. The restoration of the collector's permeability is only possible under the destruction of layered water-oil structures. As one of the possible mechanisms of destruction of such formations one can consider the parametric resonance arising under the harmonic long-time perturbation of the external boundary of a layered structure.

In [1, a system of hydrodynamical equations (in reversible and irreversible approximations) was proposed for gassy water-oil layered structures. In [2], some numerical results of the investigation of parametric instability were obtained in the framework of the model from [1].

However, already the first experimental studies of an opportunity of the organization of the parametric resonance by means of an acoustic effect specify a problem of carrying the power action into the petroliferous collectors. In this connection, it should be noted that probably it is easier to provide the power action on a layered structure by using methods of electrometry. The point is that water-oil layered structures are anisotropic

Received March 1, 2010.

2000 Mathematics Subject Classification. Primary 35Q30, 83C22.

Key words and phrases. Layered structures, anisotropic dielectrics, electrohydrodynamical approximaton, electrodynamical instability.

E-mail address: blokhin@math.nsc.ru

E-mail address: rsem86@mail.ru 
dielectrics weakly conducting the electric current. Therefore, one can state the problem on the development of parametric instability in a layered structure at the presence of a small-amplitude alternating current.

The main goal of the present work is the proof of the usual electrodynamical instability of layered structures at the presence of a small-amplitude alternating current. From the mathematical point of view, this means that this flow regime is linearly unstable (by Lyapunov). From the physical point of view, it means the practical impossibility of this flow regime.

2. Preliminaries. Let us first briefly describe the system of hydrodynamical equations (in reversible approximation) for gassy water-oil layered structures and comment on the method of obtaining it. The basis of this method is continuum approximation. In detail this approximation is described in monograph [4] and based on the validity of the conservation laws of mass, impulse, energy, and entropy. Considering a mixture of water, oil, and gas as a one-velocity layered continuum, a mathematical model of such layered structures was proposed in [1. This model consists of the following equations, which we write down in the divergent form (in the form of conservation laws):

$$
\begin{gathered}
\rho_{t}+\operatorname{div}(\rho \boldsymbol{v})=0, \\
(\rho \mathbb{C})_{t}+\operatorname{div}(\rho \mathbb{C} \boldsymbol{v})=0, \\
\left(\rho v_{i}\right)_{t}+p_{x_{i}}+\sum_{k=1}^{3}\left(\rho v_{i} v_{k}-\bar{\sigma}_{i k}\right)_{x_{k}}=0, i=1,2,3, \\
(\rho s)_{t}+\operatorname{div}(\rho s \boldsymbol{v})=0 \\
\left\{\rho\left(\varepsilon_{0}+\frac{|\boldsymbol{v}|^{2}}{2}\right)\right\}_{t}+\operatorname{div} \boldsymbol{Q}=0 .
\end{gathered}
$$

Here

$\rho$ is the density of the layered continuum,

$\boldsymbol{v}=\left(v_{1}, v_{2}, v_{3}\right)$ the medium velocity vector,

$\mathbb{C}$ the mass concentration of the gas,

$p$ the pressure,

$s$ the entropy,

$\varepsilon_{0}$ the mass internal energy, and

$|\boldsymbol{v}|^{2}=(\boldsymbol{v}, \boldsymbol{v})$.

The components $\bar{\sigma}_{i k}$ of the stress tensor and the vector $\boldsymbol{Q}=\left(Q_{1}, Q_{2}, Q_{3}\right)$ of the energy flux are described below.

To system (2.1a) - 2.1e) we add one more equation, which is obtained as follows. As the basis of the hydrodynamical model of the layered structures being considered, we can use the well-known scheme of smectics hydrodynamical description (see [5, 6]). We describe alternating layers located one after another along the $x_{3}$-axis by the explicit assignment of an allocated element of the layered structure. Such an element is a surface 
in the space with coordinates $x_{1}, x_{2}, x_{3}$ :

$$
w(\boldsymbol{x}, t)=\theta, \quad \boldsymbol{x}=\left(x_{1}, x_{2}, x_{3}\right),
$$

where $\theta$ is the parameter identifying layers. The equation of the surface can be rewritten as

$$
\boldsymbol{x}=\boldsymbol{x}(\boldsymbol{u}, t, \theta) .
$$

Here $\boldsymbol{u}=\left(u_{1}, u_{2}\right)$ are the coordinates on the surface $\theta$. Therefore,

$$
w(\boldsymbol{x}(\boldsymbol{u}, t, \theta), t) \equiv \theta
$$

and

$$
\frac{d}{d t} w=\left\{\frac{\partial}{\partial t}+(\boldsymbol{v}, \nabla)\right\} w=0
$$

is the desired equation, where

$$
\boldsymbol{v}=\left.\frac{\partial \boldsymbol{x}}{\partial t}\right|_{\boldsymbol{u}, \theta}
$$

is the velocity vector of the medium (see above). In view of equation (2.1a), expression (2.2) can be rewritten in the divergent form

$$
(\rho w)_{t}+\operatorname{div}(\rho w \boldsymbol{v})=0 .
$$

Another fundamental fact underlying continuum approximation is formulated as follows. The thermodynamical state of a nonequilibrium system is defined by external and internal parameters of this system and the energy (see [4). Also it is supposed that locally there exists a state function (nonequilibrium entropy). Therefore, to equations (2.1a) -(2.1e), (2.3), we should add the first law of thermodynamics, which for a layered structure has a complex form (see [1]):

$$
d \varepsilon_{0}=T d s-p d V+V\{(\boldsymbol{f}, d \boldsymbol{b})+Z d \mathbb{C}+\Phi d(\triangle w)+(\boldsymbol{\xi}, d \boldsymbol{a})\},
$$

where $T$ is the temperature,

$V\left(=\frac{1}{\rho}\right)$ the specific volume,

$\boldsymbol{b}=\left(b_{1}, b_{2}, b_{3}\right)=\nabla w$,

$\varepsilon_{0}=e_{0}+V E_{0}$,

$e_{0}=e_{0}(V, s, \mathbb{C})$ is the state equation,

$$
\begin{aligned}
E_{0}=\{ & \frac{B}{8}\left(\frac{|\boldsymbol{b}|^{2}}{q^{2}}-1\right)^{2}+\frac{N}{2} \frac{(\triangle w)^{2}}{q^{2}} \\
& \left.+\frac{\alpha\left(\sigma^{\prime}\right)^{2}}{2 q^{2}}|\boldsymbol{b}|^{2}\left((\boldsymbol{d}, \boldsymbol{a})^{2}-|\boldsymbol{a}|^{2}\right)+\sigma q\left(\frac{1}{d_{3}}-1\right)\right\}
\end{aligned}
$$

is the volume density of the "surface elastic energy" of a deformable layered structure (see $([1,5,6])$,

$B, N(>0), \alpha(<0)$ are some constants,

$q=q(V, s)$ is the density of the structure's layer, $\boldsymbol{d}=\left(d_{1}, d_{2}, d_{3}\right)=\frac{\boldsymbol{b}}{|\boldsymbol{b}|}$,

$\boldsymbol{a}=\left(a_{1}, a_{2}, a_{3}\right)=\nabla \mathbb{C}$, 
$\sigma=\sigma(\mathbb{C})(\geqslant 0)$ is the "surface tension",

$$
\begin{gathered}
\boldsymbol{f}=\left(f_{1}, f_{2}, f_{3}\right)=\frac{B}{2 q^{2}}\left(\frac{|\boldsymbol{b}|^{2}}{q^{2}}-1\right) \boldsymbol{b}+\frac{\alpha\left(\sigma^{\prime}\right)^{2}}{q^{2}}\left((\boldsymbol{b}, \boldsymbol{a}) \alpha-|\boldsymbol{a}|^{2} \boldsymbol{b}\right)+\frac{\sigma q}{b_{3}}\left(d_{1}, d_{2}, \frac{-d_{1}^{2}-d_{2}^{2}}{d_{3}}\right), \\
Z=\rho\left(e_{0}\right)_{\mathbb{C}}+\frac{\alpha \sigma^{\prime} \sigma^{\prime \prime}}{q^{2}}|\boldsymbol{b}|^{2}\left((\boldsymbol{d}, \boldsymbol{a})^{2}-|\boldsymbol{a}|^{2}\right)+\sigma^{\prime} q\left(\frac{1}{d_{3}}-1\right) \\
\Phi=\frac{N}{q^{2}} \triangle w
\end{gathered}
$$

$\triangle$ is the Laplace operator: $\triangle=\sum_{k=1}^{3} \frac{\partial^{2}}{\partial x_{k}^{2}}$,

$$
\begin{gathered}
\boldsymbol{\xi}=\left(\xi_{1}, \xi_{2}, \xi_{3}\right)=\frac{\alpha\left(\sigma^{\prime}\right)^{2}}{q^{2}}|\boldsymbol{b}|^{2}((\boldsymbol{d}, \boldsymbol{a}) \boldsymbol{d}-\boldsymbol{a}), \\
T=\left(e_{0}\right)_{s}+V\left(E_{0}\right)_{s}, \\
p=-\left(e_{0}\right)_{V}-E_{0}-V\left(E_{0}\right)_{V}, \\
c_{s}^{2}=p_{\rho}=-V^{2} p_{V}=-V^{2}\left\{\left(e_{0}\right)_{V V}+2\left(E_{0}\right)_{V}+V\left(E_{0}\right)_{V V}\right\}
\end{gathered}
$$

is the square of the sound velocity (see [7]),

$$
\begin{aligned}
\left(E_{0}\right)_{\beta}=-\frac{q_{\beta}}{q^{3}}\{ & \frac{B}{2}\left(\frac{|\boldsymbol{b}|^{2}}{q^{2}}-1\right)|\boldsymbol{b}|^{2}+N(\triangle w)^{2} \\
& \left.+\alpha\left(\sigma^{\prime}\right)^{2}|\boldsymbol{b}|^{2}\left((\boldsymbol{d}, \boldsymbol{a})^{2}-|\boldsymbol{a}|^{2}\right)\right\}+\sigma q_{\beta}\left(\frac{1}{d_{3}}-1\right), \quad \beta=V, s, \\
\left(E_{0}\right)_{V V}=\left(3 \frac{q_{V}^{2}}{q^{4}}-\right. & \left.\frac{q_{V V}}{q^{3}}\right)\left\{\frac{B}{2}\left(\frac{|\boldsymbol{b}|^{2}}{q^{2}}-1\right)|\boldsymbol{b}|^{2}+N(\triangle w)^{2}+\alpha\left(\sigma^{\prime}\right)^{2}|\boldsymbol{b}|^{2}\left((\boldsymbol{d}, \boldsymbol{a})^{2}-|\boldsymbol{a}|^{2}\right)\right\} \\
& +B|\boldsymbol{b}|^{4} \frac{q_{V}^{2}}{q^{6}}+\sigma q_{V V}\left(\frac{1}{d_{3}}-1\right) .
\end{aligned}
$$

REMARK 2.1. The first two terms in formula (2.5) describe the deformation of a layered structure and are intrinsic for smectic description (see [5, 6]). The next two terms are due to the dependence of the "surface tension" at the interface of the layers on the concentration $\mathbb{C}$ of the gas in the system. From the physical point of view, it is clear that the third term provides additional tangential stresses caused by the presence of gradients of the "surface tension", and the fourth term corresponds to a change in the energy of the system caused by a change in the area of the layer in the process of deformation.

Note that equations (2.1a) $-(2.1 \mathrm{~d})$ in a nonconservative form appear as follows:

$$
\begin{gathered}
\frac{d \rho}{d t}+\rho \operatorname{div} \boldsymbol{v}=0, \\
\frac{d \mathbb{C}}{d t}=0, \\
\frac{d v_{i}}{d t}+V p_{x_{i}}=V \sum_{k=1}^{3}\left(\bar{\sigma}_{i k}\right)_{x_{k}}, i=1,2,3, \\
\frac{d s}{d t}=0 .
\end{gathered}
$$


Using relations (2.6), (2.7), one can take the following equation instead of (2.8a):

$$
\frac{d p}{d t}+\rho c_{s}^{2} \operatorname{div} \boldsymbol{v}=-\left(\boldsymbol{f}+V\left(E_{0}\right)_{V \boldsymbol{b}}, \frac{d \boldsymbol{b}}{d t}\right)-\left(1-2 \frac{q_{V}}{q} V\right)\left\{\left(\boldsymbol{\xi}, \frac{d \boldsymbol{a}}{d t}\right)+\Phi \frac{d(\triangle w)}{d t}\right\} .
$$

Here

$$
\begin{aligned}
\left(E_{0}\right)_{V \boldsymbol{b}} & =\left(\left(E_{0}\right)_{V b_{1}},\left(E_{0}\right)_{V b_{2}},\left(E_{0}\right)_{V b_{3}}\right) \\
& =-\frac{q_{V}}{q^{3}}\left\{B\left(2 \frac{|\boldsymbol{b}|^{2}}{q^{2}}-1\right) \boldsymbol{b}+2 \alpha\left(\sigma^{\prime}\right)^{2}\left((\boldsymbol{b}, \boldsymbol{a}) \boldsymbol{a}-|\boldsymbol{a}|^{2} \boldsymbol{b}\right)\right\}+\frac{\sigma q_{V}}{b_{3}}\left(d_{1}, d_{2}, \frac{-d_{1}^{2}-d_{2}^{2}}{d_{3}}\right) .
\end{aligned}
$$

Moreover, by means of equations (2.2), (2.8b) the terms

$$
\frac{d \boldsymbol{b}}{d t}, \quad \frac{d \boldsymbol{a}}{d t}, \quad \frac{d(\triangle w)}{d t},
$$

appearing in the right-hand side of equation (2.9), are defined as

$$
\begin{gathered}
\frac{d \boldsymbol{b}}{d t}=-(\boldsymbol{b}, \nabla) \boldsymbol{v}-[\boldsymbol{b}, \operatorname{curl} \boldsymbol{v}], \quad \operatorname{curl} \boldsymbol{b}=0, \\
\frac{d \boldsymbol{a}}{d t}=-(\boldsymbol{a}, \nabla) \boldsymbol{v}-[\boldsymbol{a}, \operatorname{curl} \boldsymbol{v}], \quad \operatorname{curl} \boldsymbol{a}=0, \\
\frac{d(\triangle w)}{d t}=-2 \sum_{k=1}^{3}\left(\frac{\partial \boldsymbol{v}}{d x_{k}}, \nabla\right) b_{k}-(\boldsymbol{b}, \triangle \boldsymbol{v}) .
\end{gathered}
$$

Until now we did not present an explicit form of the components $\bar{\sigma}_{i k}$ of the stress tensor and the vector of energy flux $\boldsymbol{Q}$. Note that, along with the statement that conservation laws are satisfied and that the density of the internal energy depends on the thermodynamic degrees of freedom, the physical content of reversible theory is determined by the form of a stress tensor $\bar{\sigma}_{i k}$. Let us show that in reversible theory this form is uniquely determined by conservation laws (2.1a) (2.1e), (2.3), and the first law of thermodynamics (2.4). Indeed, as usual in hydrodynamics, the law of energy conservation (2.1e) should be a consequence of all the other conservation laws. Following [4, we differentiate with respect to the time the full energy

$$
E=\rho \frac{|\boldsymbol{v}|^{2}}{2}+\rho \varepsilon_{0}
$$

and, by virtue of (2.4), obtain

$$
\begin{aligned}
\frac{\partial E}{d t}=\left(\varepsilon_{0}+p V+\frac{|\boldsymbol{v}|^{2}}{2}\right) \frac{\partial \rho}{\partial t} & +\left(\rho \boldsymbol{v}, \frac{\partial \boldsymbol{v}}{\partial t}\right)+\rho T \frac{\partial s}{d t}+\left(\boldsymbol{f}, \frac{\partial \boldsymbol{b}}{\partial t}\right) \\
+ & Z \frac{\partial \mathbb{C}}{\partial t}+\Phi \frac{(\partial \triangle w)}{\partial t}+\left(\boldsymbol{\xi}, \frac{\partial \boldsymbol{a}}{\partial t}\right) .
\end{aligned}
$$

Substituting into the right-hand side of expression (2.11) the derivatives

$$
\frac{\partial \rho}{\partial t}, \quad \frac{\partial \boldsymbol{v}}{\partial t}, \quad \frac{\partial s}{\partial t}, \quad \frac{\partial \boldsymbol{b}}{\partial t}, \quad \frac{\partial \mathbb{C}}{\partial t}, \quad \frac{\partial(\triangle w)}{\partial t}, \quad \frac{\partial \boldsymbol{a}}{\partial t},
$$

which are found from (2.8a)-(2.8d), (2.10a - (2.10c), after simple but bulky calculations from (2.11) we obtain the law of energy conservation (2.1e). Doing so we define the 
vector of energy flux $\boldsymbol{Q}$ and the components $\bar{\sigma}_{i k}$ of the stress tensor as follows:

$$
\begin{gathered}
\bar{\sigma}_{i k}=b_{i}\left(-f_{k}+\frac{\partial \Phi}{\partial x_{k}}\right)-a_{i} \xi_{k}-\Phi B_{i k}, i, k=1,2,3, \\
\boldsymbol{Q}=\rho \boldsymbol{v}\left\{\varepsilon_{0}+p V+\frac{|\boldsymbol{v}|^{2}}{2}\right\}+\boldsymbol{q}, \quad \boldsymbol{q}=\left(q_{1}, q_{2}, q_{3}\right), \\
q_{k}=\left(f_{k}-\frac{\partial \Phi}{\partial x_{k}}\right)(\boldsymbol{b}, \boldsymbol{v})+\xi_{k}(\boldsymbol{a}, \boldsymbol{v})+\Phi \sum_{l=1}^{3}\left(v_{l} B_{k l}+b_{l} \frac{\partial v_{l}}{\partial x_{k}}\right), k=1,2,3,
\end{gathered}
$$

where

$$
B_{i k}=\frac{\partial^{2} w}{\partial x_{i} \partial x_{k}} .
$$

Thus, the system of conservation laws (2.1a)-(2.1d), (2.3) describes the reversible hydrodynamic motion of a layered structure. The law of energy conservation (2.1e) is a consequence of all equations of this system. The proposed ideal hydrodynamics of a layered structure can be a basis for the introduction of thermodynamic irreversibility in the motion equations (see [1]).

REMARK 2.2. In [8, 9, conditions on a surface of strong discontinuity for layered structures were obtained, a linearized stability problem for shock waves was formulated and its ill-posedness was proved (this means the instability of shock waves for the hydrodynamical model described above).

3. Hydrodynamics of an anisotropic layered dielectrics. Taking the equations of reversible hydrodynamics of layered structures described in the previous section as a basis, we show how to obtain a closed system connecting electrodynamics and mechanics of anisotropic layered dielectrics to within terms of order $\frac{1}{c^{2}}$, where $c$ is the speed of light.

First of all, we observe that processes of polarization and magnetization typical for anisotropic dielectrics imply the following modified version of the first law of thermodynamics (2.4) (see [10]):

$$
\begin{aligned}
d \varepsilon_{0}= & T d s-p d V+\left(\boldsymbol{E}^{\prime}, d \mathcal{P}\right)-\left(\boldsymbol{m}^{\prime}, d \boldsymbol{B}\right) \\
& +V\{(\boldsymbol{f}, d \boldsymbol{b})+Z d \mathbb{C}+\Phi d(\triangle w)+(\boldsymbol{\xi}, d \boldsymbol{a})\} .
\end{aligned}
$$

Here

$\varepsilon_{0}=e_{0}+V E_{0}$ is the internal energy,

$E_{0}$ is defined by (2.5),

$e_{0}=e_{0}(V, s, \mathbb{C}, \mathcal{P}, \boldsymbol{B})$ is the state equation,

$\boldsymbol{E}=\left(E_{1}, E_{2}, E_{3}\right)$ is the electric force (in the laboratory reference frame),

$\boldsymbol{E}^{\prime}=\left(\boldsymbol{E}+\frac{1}{c}[\boldsymbol{v}, \boldsymbol{B}]\right)$ is the electric force in the rest frame of a liquid particle of the medium,

$\boldsymbol{B}=\left(B_{1}, B_{2}, B_{3}\right)$ is the vector of magnetic induction (in the laboratory reference frame), $\boldsymbol{B}^{\prime}=\left(\boldsymbol{B}-\frac{1}{c}[\boldsymbol{v}, \boldsymbol{E}]\right)$ is the vector of magnetic induction in the rest frame,

$\boldsymbol{P}$ is the vector of electric polarization (in the laboratory reference frame),

$\mathcal{P}=V \boldsymbol{P}$,

$\boldsymbol{M}$ is the magnetization of the medium (in the laboratory reference frame), 
$\boldsymbol{M}^{\prime}=\left(\boldsymbol{M}+\frac{1}{c}[\boldsymbol{v}, \boldsymbol{P}]\right)$ is the magnetization of the medium in the rest frame, and $\boldsymbol{m}=V \boldsymbol{M}, \boldsymbol{m}^{\prime}=V \boldsymbol{M}^{\prime}$.

REMARK 3.1. In (3.1) it has already been taken into account that (see [10])

$$
\begin{gathered}
P^{\prime}=\boldsymbol{P}, \\
|\boldsymbol{M}| \sim \frac{1}{c},
\end{gathered}
$$

where $\boldsymbol{P}^{\prime}$ is the vector of electric polarization in the rest frame.

Let us now write down the system of the Maxwell equations at the presence of volume charges (see [10]):

$$
\begin{gathered}
\operatorname{curl} \boldsymbol{E}=-\frac{1}{c} \frac{\partial \boldsymbol{B}}{\partial t}, \quad \operatorname{curl} \boldsymbol{H}=-\frac{1}{c} \frac{\partial \boldsymbol{D}}{\partial t}+\frac{4 \pi}{c} \boldsymbol{j} \\
\operatorname{div} \boldsymbol{D}=4 \pi \rho_{e}, \quad \operatorname{div} \boldsymbol{B}=0
\end{gathered}
$$

Here

$\boldsymbol{D}=\left(D_{1}, D_{2}, D_{3}\right)=(\boldsymbol{E}+4 \pi \boldsymbol{P})$ is the vector of electric induction (in the laboratory reference frame),

$\boldsymbol{H}=\left(H_{1}, H_{2}, H_{3}\right)=(\boldsymbol{B}-4 \pi \boldsymbol{M})$ is the vector of the magnetic field (in the laboratory reference frame),

$\boldsymbol{j}=\left(j_{1}, j_{2}, j_{3}\right)$ is the vector of current density,

and $\rho_{e}$ is the volume charge density.

REMARK 3.2. The last two equations in system (3.2) can be considered as additional restrictions on the initial data. This becomes obvious if we add the law of volume charge conservation

$$
\frac{\partial \rho_{e}}{\partial t}+\operatorname{div} \boldsymbol{j}=0
$$

to 3.2 .

Before passing to the construction of the system of conservation laws connecting the electrodynamics and mechanics of layered anisotropic dielectrics, we present two relations obtained from the Maxwell equations by simple but bulky calculations. The first of them is an expression for ponderomotive forces (i.e., forces acting from the side of the electromagnetic field to a layered structure under polarization and magnetization; see $([10])$. This relation is given in the form

$$
\begin{gathered}
\frac{1}{4 \pi c}\left([\boldsymbol{E}, \boldsymbol{B}]_{i}\right)_{t}=\frac{1}{c}\left([\boldsymbol{B}, \boldsymbol{P}]_{i}\right)_{t}-\left(\boldsymbol{P}, \boldsymbol{E}_{x_{i}}\right)-\left(\boldsymbol{M}, \boldsymbol{B}_{x_{i}}\right)-\frac{1}{c}[\boldsymbol{j}, \boldsymbol{B}]_{i} \\
+\sum_{k=1}^{3}\left\{\frac{B_{k} H_{i}+D_{k} E_{i}}{4 \pi}-\left(\frac{|\boldsymbol{E}|^{2}+|\boldsymbol{B}|^{2}}{8 \pi}-(\boldsymbol{B}, \boldsymbol{M})\right) \delta_{i k}\right\}_{x_{k}}-\rho_{e} E_{i}, i=1,2,3,
\end{gathered}
$$

where $[\boldsymbol{E}, \boldsymbol{B}]_{i}$ is the $i$ th component of the vector product $[\boldsymbol{E}, \boldsymbol{B}]$, etc. The second relation is the Umow-Pointing equation (see [10]) (it describes an exchange of energy between the electromagnetic field and the medium)

$$
\frac{\partial}{\partial t}\left(\frac{|\boldsymbol{E}|^{2}+|\boldsymbol{B}|^{2}}{8 \pi}\right)=-\frac{c}{4 \pi} \operatorname{div}([\boldsymbol{E}, \boldsymbol{H}])-(\boldsymbol{j}, \boldsymbol{E})-\left(\boldsymbol{E}, \boldsymbol{P}_{t}\right)+\left(\boldsymbol{M}, \boldsymbol{B}_{t}\right) .
$$


We now begin to construct the above-mentioned system of conservation laws. Instead of equations (2.1a) $-(2.1 \mathrm{~d})$ we take

$$
\begin{gathered}
\rho_{t}+\operatorname{div}(\rho \boldsymbol{v})=0, \\
(\rho \mathbb{C})_{t}+\operatorname{div}(\rho \mathbb{C} \boldsymbol{v})=0, \\
\left\{\rho v_{i}+\frac{1}{4 \pi c}[\boldsymbol{E}, \boldsymbol{B}]_{i}\right\}_{t}+\left\{p+\frac{|\boldsymbol{E}|^{2}+|\boldsymbol{B}|^{2}}{8 \pi}-(\boldsymbol{B}, \boldsymbol{M})\right\}_{x_{i}} \\
+\sum_{k=1}^{3}\left(\rho v_{i} v_{k}-\bar{\sigma}_{i k}-\frac{H_{i} B_{k}+E_{i} D_{k}}{4 \pi}+\Sigma_{i k}\right)_{x_{k}}=0, i=1,2,3, \\
(\rho s)_{t}+\operatorname{div}(\rho s \boldsymbol{v})=\frac{R}{T} .
\end{gathered}
$$

Here $R$ is the dissipative function (in connection with the presence of the current density vector $\boldsymbol{j}$ in the Maxwell equations, we should include the entropy production in the righthand side of the law of entropy conservation); an explicit form of $\Sigma_{i k}$ and the dissipative function $R$ will be described below.

Let us calculate the time derivative of the full energy

$$
\mathcal{E}=\rho \frac{|\boldsymbol{v}|^{2}}{2}+\rho \varepsilon_{0}+\frac{|\boldsymbol{E}|^{2}+|\boldsymbol{B}|^{2}}{8 \pi} .
$$

In view of (3.1),

$$
\begin{aligned}
\frac{\partial \mathcal{E}}{\partial t}= & \frac{\partial}{\partial t}\left(\frac{|\boldsymbol{E}|^{2}+|\boldsymbol{B}|^{2}}{8 \pi}\right)+\left(\varepsilon_{0}+p V+\frac{|\boldsymbol{v}|^{2}}{2}-\left(\boldsymbol{E}^{\prime}, \mathcal{P}\right)\right) \frac{\partial \rho}{\partial t} \\
& +\left(\rho \boldsymbol{v}, \frac{\partial \boldsymbol{v}}{\partial t}\right)+\rho T \frac{\partial s}{\partial t}+\left(\boldsymbol{f}, \frac{\partial \boldsymbol{b}}{\partial t}\right)+Z \frac{\partial \mathbb{C}}{\partial t}+\Phi \frac{\partial(\triangle w)}{\partial t} \\
& +\left(\boldsymbol{\xi}, \frac{\partial a}{\partial t}\right)+\left(\boldsymbol{E}^{\prime}, \frac{\partial \boldsymbol{P}}{\partial t}\right)-\left(\boldsymbol{M}^{\prime}, \frac{\partial \boldsymbol{B}}{\partial t}\right) .
\end{aligned}
$$

Substituting the derivatives

$$
\frac{\partial \rho}{\partial t}, \quad \frac{\partial \boldsymbol{v}}{\partial t}, \quad \frac{\partial s}{\partial t}, \quad \frac{\partial \boldsymbol{b}}{\partial t}, \quad \frac{\partial \mathbb{C}}{\partial t}, \quad \frac{\partial(\triangle w)}{\partial t}, \quad \frac{\partial \boldsymbol{a}}{\partial t}
$$

found from (3.6a)-(3.6d) and (2.10a)-(2.10c) into (3.7) and using relations (3.4), (3.5), after cumbersome calculations from (3.7) we get the law of conservation of energy

$$
\frac{\partial \mathcal{E}}{\partial t}+\operatorname{div}\left(\boldsymbol{Q}-(\boldsymbol{E}, \boldsymbol{P}) \boldsymbol{v}+\frac{c[\boldsymbol{E}, \boldsymbol{H}]}{4 \pi}\right)=0
$$

if $\Sigma_{i k}$ and the dissipative function $R$ are defined as follows:

$$
\begin{gathered}
\Sigma_{i k}=[\boldsymbol{B}, \boldsymbol{P}]_{i} \frac{v_{k}}{c}, i, k=1,2,3, \\
R=\left(\left(\boldsymbol{j}-\rho_{e} \boldsymbol{v}\right), \boldsymbol{E}^{\prime}\right) .
\end{gathered}
$$

The components $\bar{\sigma}_{i k}$ of the stress tensor and the energy flux $\boldsymbol{Q}$ were defined in Sect. 1 .

The expression for the dissipative function $R$ (see (3.9) ) introduces Ohm's law for moving dielectrics with outflow:

$$
\boldsymbol{j}=\rho_{e}\left(\boldsymbol{v}+\boldsymbol{E}^{\prime} \Omega\right),
$$


where $\Omega=\left(\Omega_{i k}\right), i, k=1,2,3$ is the conductivity tensor (or the mobility tensor; see [3]), and we shall suppose that its structure corresponds to smectic symmetry (see [6]):

$$
\Omega=\omega_{\perp} I_{3}+\left(\omega_{\|}-\omega_{\perp}\right) \mathcal{D}
$$

$I_{3}$ is the unitary matrix of the third order,

$\mathcal{D}=\left(d_{i} d_{k}\right), i, k=1,2,3$,

$\omega_{\|}, \omega_{\perp}$ are some constants.

By virtue of (3.9) and (3.10), we finally get the following expression for the dissipative function $R$ :

$$
R=\rho_{e}\left(\boldsymbol{E}^{\prime} \Omega, \boldsymbol{E}^{\prime}\right),
$$

where $\Omega>0$ if $R>0$.

Thus, the system of conservation laws (3.6a) -3.6d), (2.3), (3.2) represents the full system of electrohydrodynamical equations for liquid dielectrics, and the law of energy conservation (3.8) is a consequence of this system.

On smooth solutions, the above formulated system of conservation laws can be reduced to a nonconservative form. After simple but long calculations we obtain this nonconservative form:

$$
\begin{gathered}
\frac{d \rho}{d t}+\rho \operatorname{div} \boldsymbol{v}=0 \quad\left(\text { or } \frac{d V}{d t}-V \operatorname{div} \boldsymbol{v}=0\right), \\
\frac{d w}{d t}=0, \\
\frac{d s}{d t}=\frac{R V}{T}, \\
\frac{d \mathbb{C}}{d t}=0, \\
\frac{d}{d t}\left\{v_{i}+\frac{[\boldsymbol{B}, \mathcal{P}]_{i}}{c}\right\}+V p_{x_{i}}=V \sum_{k=1}^{3}\left(\bar{\sigma}_{i k}\right)_{x_{k}}+\left(\mathcal{P}, \boldsymbol{E}_{x_{i}}\right) \\
+\left(\boldsymbol{m}, B_{x_{i}}\right)+\frac{1}{c} V[\boldsymbol{j}, \boldsymbol{B}]_{i}+\rho_{e} V E_{i}, i=1,2,3 .
\end{gathered}
$$

We note that in the motion equations (3.13e obtained from the vector law of impulse conservation there is a ponderomotive force (more precisely, its components) which at the presence of polarization and magnetization differs from the Lorentz force

$$
\rho_{e} \boldsymbol{E}+\frac{1}{c}[\boldsymbol{j}, \boldsymbol{B}] .
$$

As in Sect. 1, it follows from (3.1) that

$$
\begin{gathered}
p=-\left(e_{0}\right)_{V}-E_{0}-V\left(E_{0}\right)_{V}, \\
c_{s}^{2}=p_{\rho}=-V^{2} p_{V}=V^{2}\left\{\left(e_{0}\right)_{V V}+2\left(E_{0}\right)_{V}+V\left(E_{0}\right)_{V V}\right\} .
\end{gathered}
$$

Using these relations, we can replace (3.13a) with the equation for the pressure $p$ :

$$
\begin{gathered}
\frac{d p}{d t}+\rho c_{s}^{2} \operatorname{div} \boldsymbol{v}=p_{s} \frac{R V}{T}-\left(\boldsymbol{f}+V\left(E_{0}\right)_{V \boldsymbol{b}}, \frac{d \boldsymbol{b}}{d t}\right) \\
-\left(1-2 \frac{q_{V}}{q} V\right)\left\{\left(\boldsymbol{\xi}, \frac{d \boldsymbol{a}}{d t}\right)+\Phi \frac{d(\triangle w)}{d t}\right\}-\left(\left(e_{0}\right)_{V \mathcal{P}}, \frac{d \mathcal{P}}{d t}-\left(\left(e_{0}\right)_{V \boldsymbol{B}}, \frac{d \boldsymbol{B}}{d t}\right) .\right.
\end{gathered}
$$


Here

$$
\begin{gathered}
p_{s}=-\left(e_{0}\right)_{V s}-\left(E_{0}\right)_{s}-V\left(E_{0}\right)_{V s}, \\
\left(E_{0}\right)_{V s}=\left(3 \frac{q_{V} q_{s}}{q^{4}}-\frac{q_{V s}}{q^{3}}\right)\left\{\frac{B}{2}\left(\frac{|\boldsymbol{b}|^{2}}{q^{2}}-1\right)|\boldsymbol{b}|^{2}+N(\triangle w)^{2}+\alpha\left(\sigma^{\prime}\right)^{2}|\boldsymbol{b}|^{2}\left((\boldsymbol{d}, \boldsymbol{a})^{2}-|\boldsymbol{a}|^{2}\right)\right\} \\
+B|\boldsymbol{b}|^{4} \frac{q_{V} q_{s}}{q^{6}}+\sigma q_{V s}\left(\frac{1}{d_{3}}-1\right) .
\end{gathered}
$$

4. Mathematical model of layered dielectrics in electrohydrodynamical approximation. The mathematical model described in the previous section is rather complicated, and below we shall consider its simplified version corresponding to electrohydrodynamical approximation (see [3]) by assuming the absence of the gas phase. Let us begin with the Maxwell equations (3.2). The electric field to within terms of order $\frac{1}{c}$ satisfies the equations obtained from (3.2), (3.3), (3.10):

$$
\begin{gathered}
\operatorname{curl} \boldsymbol{E}=0, \\
\operatorname{div} \boldsymbol{D}=4 \pi \rho_{e}, \\
\frac{\partial \rho_{e}}{\partial t}+\operatorname{div} \boldsymbol{j}=0, \\
\boldsymbol{j}=\rho_{e}(\boldsymbol{v}+\boldsymbol{E} \Omega) .
\end{gathered}
$$

By virtue of Remark 2.1, in the absence of the gas phase, the first law of thermodynamics (3.1) in electrohydrodynamical approximation becomes

$$
d \varepsilon_{0}=T d s-p d V+(\boldsymbol{E}, d \mathcal{P})+V\{(\boldsymbol{f}, d \boldsymbol{b})+\Phi d(\triangle w)\} .
$$

Here $\varepsilon_{0}=e_{0}+V E_{0}$ is the internal energy, $e_{0}=e_{0}(V, s, \mathcal{P})$ the state equation,

$$
E_{0}=\left\{\frac{B}{8}\left(\frac{|\boldsymbol{b}|^{2}}{q^{2}}-1\right)^{2}+\frac{N}{2} \frac{(\triangle w)^{2}}{q^{2}}+\sigma q\left(\frac{1}{d_{3}}-1\right)\right\}
$$

the volume density of "surface elastic energy" in the absence of the gas phase, $\sigma=$ const $\geqslant 0$ the "surface tension",

$$
\boldsymbol{f}=\frac{B}{2 q^{2}}\left(\frac{|\boldsymbol{b}|^{2}}{q^{2}}-1\right) \boldsymbol{b}+\frac{\sigma q}{b_{3}}\left(d_{1}, d_{2}, \frac{-d_{1}^{2}-d_{2}^{2}}{d_{3}}\right) .
$$

Let

$$
\begin{gathered}
\varepsilon_{0}=U+(\boldsymbol{E}, \mathcal{P}), \\
U=U_{0}+V E_{0},
\end{gathered}
$$

where $U_{0}=U_{0}(V, s, \boldsymbol{E})$ is the state equation.

In view of (4.4), the thermodynamic identity (4.2) becomes

$$
d U=T d s-p d V-(\mathcal{P}, d \boldsymbol{E})+V\{(\boldsymbol{f}, d \boldsymbol{b})+\Phi d(\triangle w)\} .
$$

It follows from (4.5) that

$$
\begin{gathered}
p=-\left(U_{0}\right)_{V}-E_{0}-V\left(E_{0}\right)_{V}, \\
\mathcal{P}=-\left(U_{0}\right)_{\boldsymbol{E}}=\left(-\left(U_{0}\right)_{E_{1}},-\left(U_{0}\right)_{E_{2}},-\left(U_{0}\right)_{E_{3}}\right) .
\end{gathered}
$$

We now begin to simplify system (3.6a) - (3.6d). The main difficulty consists in the simplification of the law of impulse conservation (3.6c). In view of (3.4), we reduce it to 
(3.13e). By passing in (3.13e) to electrohydrodynamical approximation (i.e., by dropping terms of the order $\frac{1}{c}$ ) we get

$$
\frac{d v_{i}}{d t}+V p_{x_{i}}=V \sum_{k=1}^{3}\left(\bar{\sigma}_{i k}\right)_{x_{k}}+\left(\mathcal{P}, \boldsymbol{E}_{x_{i}}\right)+\rho_{e} V E_{i}, i=1,2,3 .
$$

Here $\bar{\sigma}_{i k}=b_{i}\left(-f_{k}+\Phi_{x_{k}}\right)-\Phi B_{i k}, i, k=1,2,3$ (see (2.12)). Considering (3.6a)), we rewrite (4.7) in the conservation form

$$
\left(\rho v_{i}\right)_{t}+p_{x_{i}}+\sum_{k=1}^{3}\left(\rho v_{i} v_{k}-\bar{\sigma}_{i k}\right)_{x_{k}}=\left(\boldsymbol{P}, \boldsymbol{E}_{x_{i}}\right)+\rho_{e} E_{i}, i=1,2,3 .
$$

Keeping in mind further applications (e.g., the study of the stability of shock waves), it is convenient to rewrite (4.8) as follows. In view of (4.1), we rewrite the right-hand side of (4.8):

$$
\begin{aligned}
\left(\boldsymbol{P}, \boldsymbol{E}_{x_{i}}\right)+\rho_{e} E_{i} & =\frac{\left(4 \pi \boldsymbol{P}, \boldsymbol{E}_{x_{i}}\right)+E_{i} \operatorname{div} \boldsymbol{D}}{4 \pi} \\
& =\frac{\left(\boldsymbol{D}, \boldsymbol{E}_{x_{i}}\right)+E_{i} \operatorname{div} \boldsymbol{D}-\left(\boldsymbol{E}, \boldsymbol{E}_{x_{i}}\right)}{4 \pi} \\
& =\frac{(\boldsymbol{D}, \nabla) E_{i}+E_{i} \operatorname{div} \boldsymbol{D}}{4 \pi}-\frac{1}{8 \pi}\left(|\boldsymbol{E}|^{2}\right)_{x_{i}} \\
& =\frac{\operatorname{div}\left(E_{i} \boldsymbol{D}\right)}{4 \pi}-\frac{1}{8 \pi}\left(|\boldsymbol{E}|^{2}\right)_{x_{i}} .
\end{aligned}
$$

In view of (4.9), it follows from (4.8) that

$$
\left(\rho v_{i}\right)_{t}+\left\{p+\frac{|\boldsymbol{E}|^{2}}{8 \pi}\right\}_{x_{i}}+\sum_{k=1}^{3}\left(\rho v_{i} v_{k}-\Sigma_{i k}\right)_{x_{k}}=0, i=1,2,3,
$$

where

$$
\Sigma_{i k}=\bar{\sigma}_{i k}+\frac{E_{i} D_{k}}{4 \pi} .
$$

Using the Umow-Pointing equation (3.5), we rewrite the law of energy conservation as follows:

Here

$$
\left\{\rho\left(\varepsilon_{0}+\frac{|\boldsymbol{v}|^{2}}{2}\right)\right\}_{t}+\operatorname{div} \boldsymbol{Q}=(\boldsymbol{j}, \boldsymbol{E})+\left(\boldsymbol{E}, \boldsymbol{P}_{t}\right)
$$

$$
\begin{gathered}
\boldsymbol{Q}=\rho \boldsymbol{v}\left\{U+p V+\frac{|\boldsymbol{v}|^{2}}{2}\right\}+\boldsymbol{q}, \quad \boldsymbol{q}=\left(q_{1}, q_{2}, q_{3}\right), \\
\left.q_{k}=\left(f_{k}-\frac{\partial \Phi}{\partial x_{k}}\right)(\boldsymbol{b}, \boldsymbol{v})+\Phi \sum_{l=1}^{3}\left(v_{l} B_{k l}+b_{l} \frac{\partial v_{l}}{\partial x_{k}}\right), \quad k=1,2,3 \text { (see (2.13) and (2.14) }\right) .
\end{gathered}
$$

For a further simplification of the equations of layered structures in electrohydrodynamical approximation we consider the case when the state equation

$$
U_{0}=U_{0}(V, s, \boldsymbol{E})
$$

has the form

$$
U_{0}=\tilde{U}_{0}(V, s)-\frac{V}{2}(\boldsymbol{E}, \boldsymbol{E} \Psi)
$$


where $\Psi=\Psi^{*}$ is the constant matrix of dielectric sensitivity. It follows from (4.12) that

$$
\mathcal{P}=V \boldsymbol{E} \Psi
$$

i.e.

$$
\boldsymbol{P}=\boldsymbol{E} \Psi, \quad \boldsymbol{D}=\boldsymbol{E}\left(I_{3}+4 \pi \Psi\right)=\boldsymbol{E} \tilde{\Psi} .
$$

Let

$$
U=\tilde{U}-\frac{V}{2}(\boldsymbol{E}, \boldsymbol{E} \Psi), \quad \tilde{U}=\tilde{U}_{0}+V E_{0} .
$$

Then, the thermodynamic identity (4.5) is rewritten as

$$
d \tilde{U}=T d s-\tilde{p} d V+V\{(\boldsymbol{f}, d \boldsymbol{b})+\Phi d(\triangle w)\} .
$$

Here

$$
\tilde{p}=\left(p-\frac{1}{2}(\boldsymbol{E}, \boldsymbol{E} \Psi)\right)=-\left(\tilde{U}_{0}\right)_{V}-E_{0}-V\left(E_{0}\right)_{V} .
$$

Thus, taking into account (4.12), the law of energy conservation in electrohydrodynamical approximaton takes the form

$$
\left\{\rho\left(\tilde{U}+\frac{|\boldsymbol{v}|^{2}}{2}\right)\right\}_{t}+\operatorname{div} \boldsymbol{Q}=(\boldsymbol{j}, \boldsymbol{E}),
$$

where

$$
\boldsymbol{Q}=\rho \boldsymbol{v}\left\{\tilde{U}+\tilde{p} V+\frac{|\boldsymbol{v}|^{2}}{2}\right\}+\boldsymbol{q},
$$

and the vector $\boldsymbol{q}$ is described above. The system hydrodynamic equations of layered structures in electrohydrodynamical approximation is finally written as

$$
\begin{gathered}
\rho_{t}+\operatorname{div}(\rho \boldsymbol{v})=0, \\
\left(\rho v_{i}\right)_{t}+\left\{p+\frac{|\boldsymbol{E}|^{2}}{8 \pi}\right\}_{x_{i}}+\sum_{k=1}^{3}\left(\rho v_{i} v_{k}-\Sigma_{i k}\right)_{x_{k}}=0, i=1,2,3, \\
(\rho s)_{t}+\operatorname{div}(\rho s \boldsymbol{v})=\frac{R}{T}, \\
\left\{\rho\left(\tilde{U}+\frac{|\boldsymbol{v}|^{2}}{2}\right)\right\}_{t}+\operatorname{div} \boldsymbol{Q}=(\boldsymbol{j}, \boldsymbol{E}),
\end{gathered}
$$

where $R=\rho_{e}(\boldsymbol{E} \Omega, \boldsymbol{E})$, and the components $\Sigma_{i k}$ of the stress tensor and the energy flux vector $\boldsymbol{Q}$ are described above.

Instead of system (4.16a)-(4.16d) we now have the following nonconservative form:

$$
\begin{gathered}
\frac{d \rho}{d t}+\rho \operatorname{div} \boldsymbol{v}=0, \\
\frac{d w}{d t}=0, \\
\frac{d s}{d t}=\frac{R V}{T}, \\
\frac{d v_{i}}{d t}+V \tilde{p}_{x_{i}}=V \sum_{k=1}^{3}\left(\bar{\sigma}_{i k}\right)_{x_{k}}+\rho_{e} V E_{i}, i=1,2,3 .
\end{gathered}
$$


Using relation (4.14), we can replace equation (4.17a) with the equation for $\tilde{p}$ :

$$
\frac{d \tilde{p}}{d t}+\rho c_{s}^{2} \operatorname{div} \boldsymbol{v}=\tilde{p}_{s} \frac{R V}{T}-\left(\boldsymbol{f}+V\left(E_{0}\right)_{V \boldsymbol{b}}, \frac{d \boldsymbol{b}}{d t}\right)-\left(1-2 \frac{q_{V}}{q} V\right) \Phi \frac{d(\triangle w)}{d t} .
$$

Here

$$
\begin{gathered}
c_{s}^{2}=\tilde{p}_{\rho}=-V^{2} \tilde{p}_{V}=V^{2}\left\{\left(\tilde{U}_{0}\right)_{V V}+2\left(E_{0}\right)_{V}+V\left(E_{0}\right)_{V V}\right\}, \\
\tilde{p}_{s}=-\left(\tilde{U}_{0}\right)_{V s}-\left(E_{0}\right)_{s}-V\left(E_{0}\right)_{V s},
\end{gathered}
$$

where the terms $\left(E_{0}\right)_{V},\left(E_{0}\right)_{V V},\left(E_{0}\right)_{s},\left(E_{0}\right)_{V s}$, and $\left(E_{0}\right)_{V \boldsymbol{b}}$ are found from the formulas written above by assuming that $\boldsymbol{a} \equiv 0$, and the derivatives

$$
\frac{d \boldsymbol{b}}{d t}, \frac{d(\triangle w)}{d t}
$$

are defined from (2.10a), (2.10c).

REMARK 4.1. In equations (4.17d), the influence of ponderomotive forces, i.e., the forces acting from the side of the electric field on a layered structure in which polarization is going on, is carried out through the item $V \tilde{p}_{x_{i}}$.

REMARK 4.2. The law of energy conservation (4.16d) is a consequence of all the other conservation laws. Indeed, it follows from (4.13) that

$$
\begin{gathered}
\frac{\partial}{\partial t}\left\{\rho\left(\tilde{U}+\frac{|\boldsymbol{v}|^{2}}{2}\right)\right\}=\left(\tilde{U}+\tilde{p} V+\frac{|\boldsymbol{v}|^{2}}{2}\right) \frac{\partial \rho}{\partial t}+\left(\rho \boldsymbol{v}, \frac{\partial \boldsymbol{v}}{\partial t}\right) \\
+\rho T \frac{\partial s}{\partial t}+\left(\boldsymbol{f}, \frac{\partial \boldsymbol{b}}{\partial t}\right)+\Phi \frac{\partial(\triangle w)}{\partial t} .
\end{gathered}
$$

Substituting the derivatives

$$
\frac{\partial \rho}{\partial t}, \frac{\partial \boldsymbol{v}}{\partial t}, \frac{\partial s}{\partial t}, \frac{\partial \boldsymbol{b}}{\partial t}, \frac{\partial(\triangle w)}{\partial t}
$$

into the right-hand side of expression (4.20), we finally obtain the law of energy conservation (4.16d).

5. Linearization of the equations of the electrohydrodynamical model of a layered dielectric. The mathematical model (4.1), (4.16a)-4.16d) (or (4.17a)-(4.17d)) obtained in the previous section has the following simple solution (it is called basic) when $\Psi_{33}=0$ :

$$
\begin{gathered}
\rho=\hat{\rho}, s=\hat{s}, v_{1}=0, v_{2}=0, v_{3}=0, \\
w=\hat{w}=\hat{q} z, \\
\boldsymbol{E}=\hat{\boldsymbol{E}}=\left(0,0, \hat{E}_{0} \sin (\omega t)\right), \rho_{e}=0 .
\end{gathered}
$$

Here $\hat{\rho}, \hat{s}, \hat{E}_{0}$ are some constants,

$$
\hat{q}=q(\hat{V}, \hat{s}), \hat{V}=\frac{1}{\hat{\rho}} .
$$


Linearizing system (4.17a)-4.17d) about the basic solution (5.1), one gets (small perturbations are denoted by the same letters):

$$
\begin{gathered}
\frac{\partial w}{\partial t}+\hat{q} v_{3}=0, \\
\frac{\partial s}{\partial t}=\rho_{e} \sin ^{2}(\omega t) \hat{a}, \\
\frac{\partial v_{i}}{\partial t}+\hat{V} \tilde{p}_{x_{i}}=0, i=1,2, \\
\frac{\partial v_{3}}{\partial t}+\hat{V} \hat{\beta} \tilde{p}_{x_{3}}=\frac{N}{\hat{\rho} \hat{q}} \triangle^{2} w-\frac{B}{\hat{\rho} \hat{q}} \hat{\lambda}^{2} \frac{\partial^{2} w}{\partial x_{3}^{2}}-\hat{V} \sigma \triangle_{\perp} w+\Sigma \frac{\partial s}{\partial x_{3}}+\hat{a}_{3} \rho_{e} \sin (\omega t), \\
\frac{\partial \tilde{p}}{\partial t}+\hat{\rho} \hat{c}_{s}^{2} \operatorname{div} \boldsymbol{v}-B \hat{\gamma} \frac{\partial v_{3}}{\partial x_{3}}=\hat{\tilde{p}}_{s} \hat{a} \rho_{e} \sin ^{2}(\omega t),
\end{gathered}
$$

where:

$$
\begin{gathered}
\hat{a}=\frac{\omega_{\|} \hat{E}_{0}^{2}}{\hat{\rho} \hat{T}}, \quad \hat{a}_{3}=\hat{V} \hat{E}_{0}, \quad \hat{\beta}=1-\hat{B} \hat{\gamma}, \quad \hat{B}=\frac{B}{\hat{\rho} \hat{c}_{s}^{2}}, \quad \hat{\gamma}=\gamma(\hat{\rho}, \hat{s}), \\
\gamma=\frac{\rho}{q} \frac{\partial q}{\partial \rho}, \quad \triangle_{\perp}=\frac{\partial^{2}}{\partial x_{1}^{2}}+\frac{\partial^{2}}{\partial x_{2}^{2}}, \quad \triangle=\triangle_{\perp}+\frac{\partial^{2}}{\partial x_{3}^{2}}, \\
\Sigma=\hat{B}\left\{\hat{V} \hat{\gamma}\left(\hat{\tilde{U}}_{0}\right)_{V_{S}}+\hat{\rho} \hat{x} \hat{\lambda}^{2} \hat{c}_{s}^{2}\right\}, \\
\hat{\circledast}=æ(\hat{\rho}, \hat{s}), \quad \rho æ=\frac{1}{q} \frac{\partial q}{\partial s}, \quad \hat{\lambda}^{2}=1-\hat{B} \hat{\gamma}^{2}, \\
\left(\hat{\tilde{U}}_{0}\right)_{V_{S}}=\left(\tilde{U}_{0}\right)_{V_{S}}(\hat{V}, \hat{s}), \\
\hat{\rho}^{2} \hat{c}_{s}^{2}=\left\{\left(\hat{\tilde{U}}_{0}\right)_{V V}+B \hat{\rho} \hat{\gamma}^{2}\right\}(\text { see (4.19) }), \\
\left(\hat{\tilde{U}}_{0}\right)_{V V}=\left(\tilde{U}_{0}\right)_{V V}(\hat{V}, \hat{s}), \quad \hat{p}=p-\hat{E}_{0} \sin (\omega t)\left(\Psi^{(3)}, \boldsymbol{E}\right), \Psi^{(3)}=\left(\Psi_{13}, \Psi_{23}, 0\right) .
\end{gathered}
$$

Note that while getting the linearized system $(5.2 \mathrm{a})-(5.2 \mathrm{e})$ we used the following expression, which is a linear analogue of (4.14):

$$
\tilde{p}=-\hat{\rho}^{2} \hat{c}_{s}^{2} V+\hat{\tilde{p}}_{s} s-\frac{B \hat{\gamma}}{\hat{q}} \frac{\partial w}{\partial x_{3}} .
$$

Here $\hat{\tilde{p}}_{s}=-\left(\hat{\tilde{U}}_{0}\right)_{V_{S}}+B \hat{\gamma} \hat{\rho} \hat{æ}$. Moreover, it follows from (5.3) that

$$
\hat{\lambda}^{2}=\frac{\hat{c}^{2}}{\hat{c}_{s}^{2}}=1-\hat{B} \hat{\gamma}^{2} .
$$

Here $\hat{c}^{2}=\hat{V}^{2}\left(\hat{\tilde{U}}_{0}\right)_{V V}$.

The linearization of the Maxwell equations (4.1) gives us the relations

$$
\begin{gathered}
\operatorname{curl} \boldsymbol{E}=0, \\
\operatorname{div}(\boldsymbol{E} \tilde{\Psi})=4 \pi \rho_{e}, \\
\frac{\partial \rho_{e}}{\partial t}+\hat{\xi}_{3} \frac{\partial \rho_{e}}{\partial x_{3}}=0 .
\end{gathered}
$$

Here $\hat{\xi}_{3}=\omega_{\|} \hat{E}_{0} \sin (\omega t)$.

While getting the equations (5.5a)- $15.5 \mathrm{c}$ ) we used a relation $\boldsymbol{D}=\boldsymbol{E} \tilde{\Psi}$. 
Let $\boldsymbol{E}=\nabla \varphi$. Then the equation (5.5b) could be transformed to

$$
\rho_{e}=\frac{1}{4 \pi} \triangle \varphi+\operatorname{div}(\boldsymbol{E}, \Psi) .
$$

Again coming back to the linear system (5.2a) (5.2e), it should be noted that it is more convenient to use its dimensionless version. For this purpose, we introduce new dependent and independent variables:

$$
\begin{gathered}
x_{i}^{\prime}=\frac{x_{i}}{z_{0}}, i=1,2,3, \\
w^{\prime}=\frac{w}{\hat{q} z_{0}}, s^{\prime}=\frac{s}{\hat{s}}, \\
v_{3}^{\prime}=\frac{v_{3}}{\hat{c}_{s}}, \\
t^{\prime}=\frac{\hat{c}_{s}}{z_{0}} t, p^{\prime}=\frac{p}{\hat{\rho} \hat{c}_{s}^{2}}, \rho^{\prime}=\frac{\rho}{\hat{\rho}}, V^{\prime}=\hat{\rho} V, \\
\rho_{e}^{\prime}=\rho_{e} \frac{z_{0} \hat{a}}{\hat{s} \hat{c}_{s}}=\rho_{e} z_{0} \hat{A}, \tilde{p}^{\prime}=p^{\prime}-\hat{A}_{3} \sin (\omega t)\left(\boldsymbol{\Psi}^{(3)}, \boldsymbol{E}^{\prime}\right), \boldsymbol{E}^{\prime}=\hat{A} \boldsymbol{E} .
\end{gathered}
$$

Here $z_{0}>0$ characterizes the thickness of a film, and the value $\hat{A}_{3}$ is described below. Then, system (5.2a) - (5.2e) becomes (below we drop the primes):

$$
\begin{gathered}
\frac{\partial w}{\partial t}+v_{3}=0, \\
\frac{\partial s}{\partial t}=\rho_{e} \sin ^{2}(\omega t), \\
\frac{\partial v_{i}}{\partial t}+\tilde{p}_{x_{i}}=0, i=1,2, \\
\frac{\partial v_{3}}{\partial t}+\hat{\beta} \tilde{p}_{x_{3}}=\hat{N} \triangle^{2} w-\hat{B} \hat{\lambda}^{2} \frac{\partial^{2} w}{\partial x_{3}^{2}}-\hat{\sigma} \triangle_{\perp} w+\hat{\Sigma} \frac{\partial s}{\partial x_{3}}+\hat{A}_{3} \rho_{e} \sin (\omega t), \\
\frac{\partial \tilde{p}}{\partial t}+\operatorname{div}_{\perp} \boldsymbol{v}+\hat{\beta} \frac{\partial v_{3}}{\partial x_{3}}=R_{1} \rho_{e} \sin ^{2}(\omega t),
\end{gathered}
$$

where

$$
\begin{gathered}
\hat{A}_{3}=\frac{1}{\hat{\Lambda}} \frac{\hat{s} T}{\hat{c}_{s}^{2}}, \hat{\Lambda}=\frac{\omega_{\|} \hat{E}_{0}}{\hat{c}_{s}}, \\
\hat{N}=\frac{N}{\hat{\rho} \hat{c}_{s}^{2} z_{0}^{2}}, \hat{\sigma}=\frac{\sigma \hat{q}}{\hat{\rho} \hat{c}_{s}^{2}}, \hat{\Sigma}=\frac{\Sigma \hat{s}}{\hat{c}_{s}^{2}} \\
\operatorname{div}_{\perp} \boldsymbol{v}=\frac{\partial v_{1}}{\partial x_{1}}+\frac{\partial v_{2}}{\partial x_{2}}, R_{1}=\frac{\hat{s}\left\{B \hat{\gamma} \hat{\rho} \hat{\circledast}-\left(\hat{\tilde{U}}_{0}\right)_{V_{s}}\right\}}{\hat{\rho} \hat{c}_{s}^{2}} .
\end{gathered}
$$

Relation (5.4) in a dimensionless form appears as follows:

$$
\tilde{p}=-V+R_{1} s-\hat{B} \hat{\gamma} \frac{\partial w}{\partial x_{3}} .
$$


The linearized Maxwell equations (5.5a - (5.5c) in a dimensionless form are

$$
\begin{gathered}
\operatorname{curl} \boldsymbol{E}=0, \\
\rho_{e}=\frac{1}{4 \pi} \Delta \varphi+\operatorname{div}(\boldsymbol{E}, \Psi), \\
\frac{\partial \rho_{e}}{\partial t}+\hat{\Lambda} \sin (\omega t) \frac{\partial \rho_{e}}{\partial x_{3}}=0 .
\end{gathered}
$$

From equations (5.6d), (5.6e), (5.8c) we can easily obtain the following system:

$$
\begin{gathered}
\left(v_{3}\right)_{t t}-\hat{\sigma} \triangle_{\perp} v_{3}-\hat{B} \hat{\lambda}^{2}\left(v_{3}\right)_{x_{3} x_{3}}+\hat{N} \triangle^{2} v_{3}+\hat{\beta} \tilde{p}_{t x_{3}} \\
=\hat{\Sigma} \sin ^{2}(\omega t)\left(\rho_{e}\right)_{x_{3}}+\hat{A}_{3} \sin (\omega t)\left(\rho_{e}\right)_{t}+\rho_{e} \hat{A}_{3} \omega \cos (\omega t), \\
\tilde{p}_{t t}-\triangle_{\perp} \tilde{p}+\hat{\beta}\left(v_{3}\right)_{t x_{3}}=R_{1}\left(\rho_{e}\right)_{t} \sin ^{2}(\omega t)+2 R_{1} \rho_{e} \sin (\omega t) \cos (\omega t) \omega, \\
\left(\rho_{e}\right)_{t}+\hat{\Lambda} \sin (\omega t)\left(\rho_{e}\right)_{x_{3}}=0 .
\end{gathered}
$$

Let's consider the Cauchy problem for the system (5.9) with the initial data:

$$
\begin{gathered}
\left.v_{3}\right|_{t=0}=v_{30}(\boldsymbol{x}),\left.\quad \frac{\partial v_{3}}{\partial t}\right|_{t=0}=v_{31}(\boldsymbol{x}), \\
\left.\tilde{p}\right|_{t=0}=\tilde{p}_{0}(\boldsymbol{x}),\left.\quad \frac{\partial \tilde{p}}{\partial t}\right|_{t=0}=\tilde{p}_{1}(\boldsymbol{x}), \\
\left.\rho_{e}\right|_{t=0}=\rho_{e 0}(\boldsymbol{x}) .
\end{gathered}
$$

Let us apply the Fourier transform to (5.9):

$$
\begin{gathered}
\hat{\rho}_{e}(t, \boldsymbol{\omega})=\int_{\mathbb{R}^{3}} e^{-2 \pi i(\boldsymbol{x}, \boldsymbol{\xi})} \rho_{e}(t, \boldsymbol{x}) d \boldsymbol{x}, \boldsymbol{\omega}=2 \pi \boldsymbol{\xi}, \hat{\rho}_{e 0}(\boldsymbol{\omega})=\int_{\mathbb{R}^{3}} e^{-2 \pi i(\boldsymbol{x}, \boldsymbol{\xi})} \rho_{e 0}(\boldsymbol{x}) d \boldsymbol{x}, \\
\hat{v}_{3}(t, \boldsymbol{\omega})=\int_{\mathbb{R}^{3}} e^{-2 \pi i(\boldsymbol{x}, \boldsymbol{\xi})} v_{3}(t, \boldsymbol{x}) d \boldsymbol{x} \\
\hat{v}_{30}(\boldsymbol{\omega})=\int_{\mathbb{R}^{3}} e^{-2 \pi i(\boldsymbol{x}, \boldsymbol{\xi})} v_{30}(\boldsymbol{x}) d \boldsymbol{x}, \hat{v}_{31}(\boldsymbol{\omega})=\int_{\mathbb{R}^{3}} e^{-2 \pi i(\boldsymbol{x}, \boldsymbol{\xi})} v_{31}(\boldsymbol{x}) d \boldsymbol{x}, \\
\hat{\tilde{p}}(t, \boldsymbol{\omega})=\int_{\mathbb{R}^{3}} e^{-2 \pi i(\boldsymbol{x}, \boldsymbol{\xi})} \tilde{p}(t, \boldsymbol{x}) d \boldsymbol{x}, \\
\hat{\tilde{p}}_{0}(\boldsymbol{\omega})=\int_{\mathbb{R}^{3}} e^{-2 \pi i(\boldsymbol{x}, \boldsymbol{\xi})} \tilde{p}_{0}(\boldsymbol{x}) d \boldsymbol{x}, \hat{\tilde{p}}_{1}(\boldsymbol{\omega})=\int_{\mathbb{R}^{3}} e^{-2 \pi i(\boldsymbol{x}, \boldsymbol{\xi})} \tilde{p}_{1}(\boldsymbol{x}) d \boldsymbol{x} .
\end{gathered}
$$

Then, system (5.9) implies

$$
\begin{gathered}
\hat{\rho}_{e}^{\prime}+\hat{\Lambda} \sin (\omega t) i \omega_{3} \hat{\rho}_{e}=0 \text { or } \\
\hat{\rho}_{e}=\hat{\rho}_{e}(t, \boldsymbol{\omega})=\hat{\rho}_{e 0}(\boldsymbol{\omega}) \exp \left\{\frac{\cos (\omega t)-1}{\omega} i \omega_{3} \hat{\Lambda}\right\}, \\
\hat{v}_{3}^{\prime \prime}+\left(\hat{\sigma}\left|\boldsymbol{\omega}_{\perp}\right|^{2}+\hat{B} \hat{\lambda}^{2} \omega_{3}^{2}+\hat{N}|\boldsymbol{\omega}|^{4}\right) \hat{v}_{3}+i \omega_{3} \hat{\beta} \hat{\tilde{p}}^{\prime} \\
=\hat{\Sigma}_{1} \sin ^{2}(\omega t) i \omega_{3} \hat{\rho}_{e}+\hat{\rho}_{e} \hat{A}_{3} \omega \cos (\omega t), \\
\hat{\tilde{p}}^{\prime \prime}+\left|\boldsymbol{\omega}_{\perp}\right|^{2} \hat{\tilde{p}}+\hat{\beta} i \omega_{3} \hat{v}_{3}^{\prime}=-R_{1} \hat{\Lambda} \sin ^{3}(\omega t) i \omega_{3} \hat{\rho}_{e}+R_{1} \hat{\rho}_{e} \omega \sin (2 \omega t),
\end{gathered}
$$


where

$$
\begin{gathered}
\left|\boldsymbol{\omega}_{\perp}\right|^{2}=\omega_{1}^{2}+\omega_{2}^{2},|\boldsymbol{\omega}|^{2}=\omega_{1}^{2}+\omega_{2}^{2}+\omega_{3}^{2}, \boldsymbol{\omega}=\left(\omega_{1}, \omega_{2}, \omega_{3}\right), \boldsymbol{\omega}_{\perp}=\left(\omega_{1}, \omega_{2}\right), \\
\hat{\Sigma}_{1}=\hat{\Sigma}-\frac{\hat{s} \hat{T}}{\hat{c}_{s}^{2}} .
\end{gathered}
$$

We can also obtain equations (5.11a) -(5.11c) by seeking for particular solutions of system (5.9) in the form

$$
\begin{aligned}
v_{3}(t, \boldsymbol{x}) & =\exp (i(\boldsymbol{\omega}, \boldsymbol{x})) \hat{v}_{3}(t), \\
\tilde{p}(t, \boldsymbol{x}) & =\exp (i(\boldsymbol{\omega}, \boldsymbol{x})) \hat{\tilde{p}}(t), \\
\rho_{e}(t, \boldsymbol{x}) & =\exp (i(\boldsymbol{\omega}, \boldsymbol{x})) \hat{\rho}_{e}(t) .
\end{aligned}
$$

First of all, let us consider the following special cases when we can find a solution of problem (5.11a) $-(5.11 \mathrm{c})$ in an explicit form:

a) $\left|\boldsymbol{\omega}_{\perp}\right|=\mu_{\perp} \neq 0, \omega_{3}=0$;

b) $\mu_{\perp}=0, \omega_{3} \neq 0$.

In case a) we have

$$
\hat{\rho}_{e}(t)=C_{\rho}=\text { const }
$$

$$
\begin{gathered}
\hat{v}_{3}^{\prime \prime}+\hat{\mu}^{2} \hat{v}_{3}=\hat{A}_{3} C_{\rho} \omega \cos (\omega t), \hat{\mu}^{2}=\mu_{\perp}^{2}\left(\hat{\sigma}+\hat{N} \mu_{\perp}^{2}\right), \\
\hat{\tilde{p}}^{\prime \prime}+\mu_{\perp}^{2} \hat{\tilde{p}}=R_{1} C_{\rho} \omega \sin (2 \omega t) .
\end{gathered}
$$

If $\omega=\hat{\mu}=\mu_{\perp} \sqrt{\hat{\sigma}+\hat{N} \mu_{\perp}^{2}}$, then equation (5.13) has the particular solution

$$
\hat{v}_{3 r}(t)=M t \sin (\hat{\mu} t), M=\text { const. }
$$

Let us define a constant $M$ by substituting the particular solution into equation (5.13):

$$
2 M \hat{\mu} \cos (\hat{\mu} t)=\hat{A}_{3} C_{\rho} \hat{\mu} \cos (\hat{\mu} t) .
$$

Then

$$
M=\frac{\hat{A}_{3} C_{\rho}}{2} .
$$

Thus, we find the general solution of (5.13) as the sum of the general solution of the homogeneous equation and the particular solution of the inhomogeneous equation:

$$
\hat{v}_{3}(t)=C_{1} \cos (\hat{\mu} t)+C_{2} \sin (\hat{\mu} t)+\frac{\hat{A}_{3} C_{\rho} t}{2} \sin (\hat{\mu} t),
$$

where $C_{1}, C_{2}$ are some constants.

Likewise, equation (5.14) has the particular solution

$$
\hat{\tilde{p}}_{r}(t)=-\frac{R_{1} C_{\rho} t}{4} \cos \left(\mu_{\perp} t\right),
$$

for the case $\omega=\frac{\mu_{\perp}}{2}$. Therefore, the general solution of (5.14) is

$$
\hat{\tilde{p}}(t)=C_{3} \cos \left(\mu_{\perp} t\right)+C_{4} \sin \left(\mu_{\perp} t\right)-\frac{R_{1} C_{\rho} t}{4} \cos \left(\mu_{\perp} t\right),
$$

where $C_{3}, C_{4}$ are some constants. 
REMARK 5.1. If $\mu_{\perp} \sqrt{\hat{\sigma}+\hat{N} \mu_{\perp}^{2}}=\frac{\mu_{\perp}}{2}$,i.e., for $\mu_{\perp}^{2}=\left(\frac{1}{4}-\hat{\sigma}\right) \frac{1}{\hat{N}}, \hat{\sigma}<\frac{1}{4}$, then

$$
\lim _{t \rightarrow \infty}\left|\hat{v}_{3}(t)\right|=\infty, \lim _{t \rightarrow \infty}|\hat{\tilde{p}}(t)|=\infty
$$

In case b) we have:

$$
\begin{gathered}
\hat{\rho}_{e}=C_{\rho} \exp \left\{\frac{\cos (\omega t)-1}{\omega} i \omega_{3} \hat{\Lambda}\right\} \\
\hat{v}_{3}^{\prime \prime}+\hat{\nu}^{2} \hat{v}_{3}+i \omega_{3} \hat{\beta} \hat{\tilde{p}}^{\prime}=\hat{\Sigma}_{1} \sin ^{2}(\omega t) i \omega_{3} \hat{\rho}_{e}+\hat{\rho}_{e} \hat{A}_{3} \omega \cos (\omega t) \\
\hat{\nu}^{2}=\omega_{3}^{2}\left(\hat{B} \hat{\lambda}^{2}+\hat{N} \omega_{3}^{2}\right) \\
\hat{\tilde{p}}^{\prime \prime}+i \omega_{3} \hat{\beta} \hat{v}_{3}^{\prime}=\left(R_{1} \hat{\rho}_{e} \sin ^{2}(\omega t)\right)^{\prime}
\end{gathered}
$$

Integrating (5.18) we obtain

$$
\hat{\tilde{p}}^{\prime}+i \omega_{3} \hat{\beta} \hat{v}_{3}=R_{1} \hat{\rho}_{e} \sin ^{2}(\omega t)+\hat{C}_{3},
$$

where $\hat{C}_{3}$ is an arbitrary constant.

Substituting the expression for $\hat{\tilde{p}}^{\prime}$ into (5.17), we get

$$
\hat{v}_{3}^{\prime \prime}+\hat{\nu}^{2} \hat{v}_{3}=F(\cos (\omega t))
$$

where

$$
\begin{gathered}
\hat{\nu}^{2}=\omega_{3}^{2}\left(\hat{B} \hat{\lambda}^{2}+\hat{N} \omega_{3}^{2}+\hat{\beta}^{2}\right), \\
F(\cos (\omega t))=\hat{\rho}_{e} \hat{A}_{3} \omega \cos (\omega t)+\hat{\Sigma}_{1} \sin ^{2}(\omega t) i \omega_{3} \hat{\rho}_{e}-i \omega_{3} \hat{\beta} \hat{C}_{3}-i \omega_{3} \hat{\beta} R_{1} \hat{\rho}_{e} \sin ^{2}(\omega t) .
\end{gathered}
$$

Let $\hat{\nu}=\omega$. As in case a), we find a general solution of (5.19):

$$
\begin{gathered}
\hat{v}_{3}(t)=C_{1} \cos (\hat{\nu} t)+C_{2} \sin (\hat{\nu} t) \\
+\frac{\sin (\hat{\nu} t)}{\hat{\nu}} \int_{0}^{t} F(\cos (\hat{\nu} \tau)) \cos (\hat{\nu} \tau) d \tau-\frac{\cos (\hat{\nu} t)}{\hat{\nu}} \int_{0}^{t} F(\cos (\hat{\nu} \tau)) \sin (\hat{\nu} \tau) d \tau .
\end{gathered}
$$

Let us expand the function $F(z)(z=\cos (\hat{\nu} t))$ in power series

$$
\begin{gathered}
F(z)=\sum_{k=0}^{\infty} a_{k} z^{k}=C_{\rho} e^{-i \hat{h}}\left(-\hat{d}+\hat{\chi}+\left(\omega \hat{A}_{3}+\hat{\chi} i \hat{h}\right) z+\sum_{k=2}^{\infty}\left[\frac{\hat{\chi}}{k !}(i \hat{h})^{k}\right.\right. \\
\left.\left.-\frac{\hat{\chi}}{(k-2) !}(i \hat{h})^{k-2}+\frac{\omega \hat{A}_{3}}{(k-1) !}(i \hat{h})^{k-1}\right] z^{k}\right)
\end{gathered}
$$

where $\hat{h}=\frac{\omega_{3}}{\omega} \hat{\Lambda}, \hat{\chi}=i \omega_{3}\left(\hat{\Sigma}_{1}-R_{1} \hat{\beta}\right), \hat{d}=i \omega_{3} \hat{\beta} \frac{C_{3}}{C_{\rho}} e^{\hat{h} i}$. We now prove that $\lim _{t \rightarrow \infty}\left|\hat{v}_{3}(t)\right|=$ $\infty$. For this purpose we consider the integrals

$$
\int_{0}^{t} F(\cos (\hat{\nu} \tau)) \sin (\hat{\nu} \tau) d \tau \text { and } \int_{0}^{t} F(\cos (\hat{\nu} \tau)) \cos (\hat{\nu} \tau) d \tau
$$

in detail. It follows from (5.21) that

$$
\begin{gathered}
\int_{0}^{t} F(\cos (\hat{\nu} \tau)) \sin (\hat{\nu} \tau) d \tau=\sum_{k=0}^{\infty} a_{k} \int_{0}^{t} \cos ^{k}(\hat{\nu} \tau) \sin (\hat{\nu} \tau) d \tau \\
=\sum_{k=0}^{\infty} a_{k}\left(1-\frac{1}{\hat{\nu}(k+1)}\right)\left(\cos ^{k+1}(\hat{\nu} t)-1\right)
\end{gathered}
$$


Consider the second integral:

$$
\int_{0}^{t} F(\cos (\hat{\nu} \tau)) \cos (\hat{\nu} \tau) d \tau=\sum_{k=0}^{\infty} a_{k} \int_{0}^{t} \cos ^{k+1}(\hat{\nu} \tau) d \tau
$$

After the integration this sum contains terms depending linearly on $t$. For studying the behavior of $\hat{v}_{3}(t)$ as $t \rightarrow \infty$ it is enough to consider only these terms. Omitting the terms which do not depend linearly on $t$, we get

$$
\begin{gathered}
\int_{0}^{t} F(\cos (\hat{\nu} \tau)) \cos (\hat{\nu} \tau) d \tau=\ldots+\left\{\omega \hat{A}_{3} \sum_{m=0}^{\infty} \frac{(-1)^{m}(\hat{h})^{2 m}}{(2 m) !} \frac{(2 m+1)(2 m-1) \ldots 1}{\hat{\nu}(2 m+2) 2 m \ldots 2}\right. \\
\left.+i\left(\frac{\hat{\chi} \hat{h}}{2}+\sum_{m=1}^{\infty}\left(\frac{(-1)^{m} \hat{h}^{2 m+1}}{(2 m+1) !}+\frac{(-1)^{m} \hat{h}^{2 m-1}}{(2 m-1) !}\right) \hat{\chi} \frac{(2 m+1)(2 m-1) \ldots 1}{\hat{\nu}(2 m+2) 2 m \ldots 2}\right)\right\} t .
\end{gathered}
$$

It is easy to check that the power series in this expression converge for any $\hat{h}$. That is, $\lim _{t \rightarrow \infty}\left|\hat{v}_{3}(t)\right|=\infty$.

Thus, in cases a) and b), the general solution for layered structures under certain conditions is unstable in the linear approximation; i.e., this solution is finally destroyed.

Now let us consider the general case. We use the new notation

$$
\begin{gathered}
\mu^{2}=\left|\omega_{\perp}\right|^{2}, \nu^{2}=\hat{\sigma}\left|\omega_{\perp}\right|^{2}+\hat{B} \hat{\lambda}^{2} \omega_{3}^{2}+\hat{N}|\boldsymbol{\omega}|^{4}, \hat{\beta}=\tilde{r} \hat{\Lambda}^{2}, \tilde{r} \text { is a constant } \\
\mathcal{A}_{3}=\hat{A}_{3} \hat{\Lambda}=\frac{\hat{s} \hat{T}}{\hat{c}_{s}^{2}}, \mathcal{V}=\hat{\Lambda} \hat{v}_{3}, \mathcal{P}=\hat{\tilde{p}}
\end{gathered}
$$

Then equations (5.11b)-(5.11c) take the form

$$
\begin{aligned}
\mathcal{P}^{\prime \prime}+\mu^{2} \mathcal{P}+i \omega_{3} \hat{\Lambda} \tilde{r} \mathcal{V}^{\prime} & =R_{1} \hat{\rho}_{e} \omega \sin (2 \omega t)-R_{1} \hat{\rho}_{e} i \omega_{3} \hat{\Lambda} \sin ^{3}(\omega t), \\
\mathcal{V}^{\prime \prime}+\nu^{2} \mathcal{V}+i \omega_{3} \tilde{r} \mathcal{P}^{\prime} & =\hat{\rho}_{e} \mathcal{A}_{3} \omega \cos (\omega t)+\hat{\rho}_{e} \hat{\Sigma}_{1} i \omega_{3} \hat{\Lambda} \sin ^{2}(\omega t)
\end{aligned}
$$

Let us seek for solutions in the following form:

$$
\begin{aligned}
& \mathcal{P}(t, \omega)=\mathcal{P}^{(0)}(t, \omega)+\mathcal{P}^{(1)}(t, \omega), \\
& \mathcal{V}(t, \omega)=\mathcal{V}^{(0)}(t, \omega)+\mathcal{V}^{(1)}(t, \omega),
\end{aligned}
$$

where the new functions are the solutions of the Cauchy problem

$$
\begin{aligned}
\mathcal{P}^{(0) \prime \prime}+\mu^{2} \mathcal{P}^{(0)}+i \omega_{3} \hat{\Lambda} \tilde{r} \mathcal{V}^{(0) \prime} & =R_{1} \hat{\rho}_{e 0} \omega \sin (2 \omega t), \\
\mathcal{V}^{(0) \prime \prime}+\nu^{2} \mathcal{V}^{(0)}+i \omega_{3} \tilde{r} \mathcal{P}^{(0) \prime} & =\hat{\rho}_{e 0} \mathcal{A}_{3} \omega \cos (\omega t)
\end{aligned}
$$

with the initial data

$$
\begin{gathered}
\left.\mathcal{P}^{(0)}\right|_{t=0}=\hat{\tilde{p}}_{0}(\boldsymbol{\omega}),\left.\quad \mathcal{P}^{(0) \prime}\right|_{t=0}=\hat{\tilde{p}}_{1}(\boldsymbol{\omega}) \\
\left.\mathcal{V}^{(0)}\right|_{t=0}=\hat{v}_{30}(\boldsymbol{\omega}),\left.\quad \mathcal{V}^{(0) \prime}\right|_{t=0}=\hat{v}_{31}(\boldsymbol{\omega})
\end{gathered}
$$


and the Cauchy problem

$$
\begin{gathered}
\mathcal{P}^{(1) \prime \prime}+\mu^{2} \mathcal{P}^{(1)}+i \omega_{3} \hat{\Lambda} \tilde{r} \mathcal{V}^{(1) \prime}=R_{1}\left(\hat{\rho}_{e}-\hat{\rho}_{e 0}\right) \omega \sin (2 \omega t) \\
-R_{1} \hat{\rho}_{e} i \omega_{3} \hat{\Lambda} \sin ^{3}(\omega t)=\hat{\Lambda} \hat{f} \\
\mathcal{V}^{(1) \prime \prime}+\nu^{2} \mathcal{V}^{(1)}+i \omega_{3} \tilde{r} \mathcal{P}^{(1) \prime}=\left(\hat{\rho}_{e}-\hat{\rho}_{e 0}\right) \mathcal{A}_{3} \omega \cos (\omega t) \\
+\hat{\rho}_{e} \hat{\Sigma}_{1} i \omega_{3} \hat{\Lambda} \sin ^{2}(\omega t)=\hat{\Lambda} \hat{g}
\end{gathered}
$$

with the initial data

$$
\begin{aligned}
& \left.\mathcal{P}^{(1)}\right|_{t=0}=\left.\mathcal{P}^{(1) \prime}\right|_{t=0}=0, \\
& \left.\mathcal{V}^{(1)}\right|_{t=0}=\left.\mathcal{V}^{(1) \prime}\right|_{t=0}=0 .
\end{aligned}
$$

We first consider problem (5.24), (5.25). Assume that the solution of this problem exists and has the form of the series

$$
\begin{aligned}
& \mathcal{P}^{(0)}=\mathcal{P}_{0}^{(0)}+\hat{\Lambda} \mathcal{P}_{1}^{(0)}+\ldots \\
& \mathcal{V}^{(0)}=\mathcal{V}_{0}^{(0)}+\hat{\Lambda} \mathcal{V}_{1}^{(0)}+\ldots
\end{aligned}
$$

Assuming that $\hat{\Lambda}$ is small and neglecting the values of order $\hat{\Lambda}^{2}$, we obtain that the functions $\mathcal{P}_{0}^{(0)}, \mathcal{P}_{1}^{(0)}, \mathcal{V}_{0}^{(0)}$, and $\mathcal{V}_{1}^{(0)}$ are the solutions of the Cauchy problem

$$
\begin{gathered}
\mathcal{P}_{0}^{(0) \prime \prime}+\mu^{2} \mathcal{P}_{0}^{(0)}=R_{1} \hat{\rho}_{e 0} \omega \sin (2 \omega t), \\
\mathcal{V}_{0}^{(0) \prime \prime}+\nu^{2} \mathcal{V}_{0}^{(0)}=\mathcal{A}_{3} \hat{\rho}_{e 0} \omega \cos (\omega t), \\
\mathcal{P}_{1}^{(0) \prime \prime}+\mu^{2} \mathcal{P}_{1}^{(0)}+i \omega_{3} \tilde{r} \mathcal{V}_{0}^{(0) \prime}=0, \\
\mathcal{V}_{1}^{(0) \prime \prime}+\nu^{2} \mathcal{V}_{1}^{(0)}=0, \\
\left.\mathcal{P}_{0}^{(0)}\right|_{t=0}=\hat{\tilde{p}}_{0}(\boldsymbol{\omega}),\left.\quad \mathcal{P}_{0}^{(0) \prime}\right|_{t=0}=\hat{\tilde{p}}_{1}(\boldsymbol{\omega}), \\
\left.\mathcal{V}_{0}^{(0)}\right|_{t=0}=\hat{v}_{30}(\boldsymbol{\omega}),\left.\quad \mathcal{V}_{0}^{(0) \prime}\right|_{t=0}=\hat{v}_{31}(\boldsymbol{\omega}), \\
\left.\mathcal{P}_{1}^{(0)}\right|_{t=0}=\left.\mathcal{P}_{1}^{(0) \prime}\right|_{t=0}=\left.\mathcal{V}_{1}^{(0)}\right|_{t=0}=\left.\mathcal{V}_{1}^{(0) \prime}\right|_{t=0}=0 .
\end{gathered}
$$

Clearly, it follows from this problem that $\mathcal{V}_{1}^{(0)}=0$.

We now consider other equations in detail.

1)

$$
\mathcal{P}_{0}^{(0) \prime \prime}+\mu^{2} \mathcal{P}_{0}^{(0)}=R_{1} \hat{\rho}_{e 0} \omega \sin (2 \omega t) .
$$

We have two cases. For the case a) $2 \omega=\mu$ (resonant case). This equation has the particular solution

$$
\mathcal{P}_{0}^{(0)}(t)=M t \cos (\mu t), M=\text { const } .
$$

Substituting this solution into the above equation, we get

$$
M=-\frac{R_{1} \hat{\rho}_{e 0}}{4} .
$$

Thus, the general solution is

$$
\mathcal{P}_{0}^{(0)}(t)=C_{1} \cos (\mu t)+C_{2} \sin (\mu t)-\frac{R_{1} \hat{\rho}_{e 0} t}{4} \cos (\mu t),
$$


where $C_{1}, C_{2}$ are some constants. Taking into account the initial data, we find these constants:

$$
\begin{gathered}
C_{1}=\hat{\tilde{p}}_{0}(\boldsymbol{\omega}), \\
C_{2}=\frac{4 \hat{\tilde{p}}_{1}(\boldsymbol{\omega})+R_{1} \hat{\rho}_{e 0}}{4 \mu} .
\end{gathered}
$$

Therefore, the exact solution of problem (5.28) for $\mathcal{P}_{0}^{(0)}$ has the form

$$
\begin{gathered}
\mathcal{P}_{0}^{(0)}(t)=\hat{\tilde{p}}_{0}(\boldsymbol{\omega}) \cos (\mu t)+\frac{4 \hat{\tilde{p}}_{1}(\boldsymbol{\omega})+R_{1} \hat{\rho}_{e 0}}{4 \mu} \sin (\mu t) \\
-\frac{R_{1} \hat{\rho}_{e 0} t}{4} \cos (\mu t) .
\end{gathered}
$$

Consider case b) $2 \omega \neq \mu$ (nonresonant case). Then the equation has the particular solution

$$
\mathcal{P}_{0}^{(0)}(t)=M \sin (2 \omega t), M=\text { const } .
$$

Substituting this solution into the equation, we get

$$
M=\frac{R_{1} \hat{\rho}_{e 0} \omega}{\mu^{2}-4 \omega^{2}} .
$$

Hence, the general solution has the form

$$
\mathcal{P}_{0}^{(0)}(t)=C_{1} \cos (\mu t)+C_{2} \sin (\mu t)+\frac{R_{1} \hat{\rho}_{e 0} \omega}{\mu^{2}-4 \omega^{2}} \sin (2 \omega t),
$$

where $C_{1}, C_{2}$ are some constants. Taking into account the initial data, we find these constants:

$$
\begin{gathered}
C_{1}=\hat{\tilde{p}}_{0}(\boldsymbol{\omega}), \\
C_{2}=\frac{\hat{\tilde{p}}_{1}(\boldsymbol{\omega})\left(\mu^{2}-4 \omega^{2}\right)-2 \omega^{2} R_{1} \hat{\rho}_{e 0}}{\mu\left(\mu^{2}-4 \omega^{2}\right)} .
\end{gathered}
$$

Therefore, the solution of problem (5.28) for $\mathcal{P}_{0}^{(0)}$ is

$$
\begin{gathered}
\mathcal{P}_{0}^{(0)}(t)=\hat{\tilde{p}}_{0}(\boldsymbol{\omega}) \cos (\mu t)+\frac{\hat{\tilde{p}}_{1}(\boldsymbol{\omega})\left(\mu^{2}-4 \omega^{2}\right)-2 \omega^{2} R_{1} \hat{\rho}_{e 0}}{\mu\left(\mu^{2}-4 \omega^{2}\right)} \sin (\mu t) \\
+\frac{R_{1} \hat{\rho}_{e 0} \omega}{\mu^{2}-4 \omega^{2}} \sin (2 \omega t) .
\end{gathered}
$$

2) Let us now consider the next equation in (5.28):

$$
\mathcal{V}_{0}^{(0) \prime \prime}+\nu^{2} \mathcal{V}_{0}^{(0)}=\mathcal{A}_{3} \hat{\rho}_{e 0} \omega \cos (\omega t)
$$

Omitting detailed calculations which are really similar to the above ones, we find the solution of this equation to be

$$
\mathcal{V}_{0}^{(0)}(t)=\hat{v}_{30}(\boldsymbol{\omega}) \cos (\nu t)+\frac{\hat{v}_{31}(\boldsymbol{\omega})}{\nu} \sin (\nu t)+\frac{\mathcal{A}_{3} \hat{\rho}_{e 0} t}{2} \sin (\nu t)
$$


for the resonant case a) $\nu=\omega$ and

$$
\begin{gathered}
\mathcal{V}_{0}^{(0)}(t)=\frac{\hat{v}_{30}(\boldsymbol{\omega})\left(\nu^{2}-\omega^{2}\right)-\mathcal{A}_{3} \hat{\rho}_{e 0} \omega}{\nu^{2}-\omega^{2}} \cos (\nu t) \\
+\frac{\hat{v}_{31}(\boldsymbol{\omega})}{\nu} \sin (\nu t)+\frac{\mathcal{A}_{3} \hat{\rho}_{e 0} \omega}{\nu^{2}-\omega^{2}} \cos (\omega t)
\end{gathered}
$$

for the nonresonant case b) $\nu \neq \omega$.

3) It remains to consider the last equation:

$$
\mathcal{P}_{1}^{(0) \prime \prime}+\mu^{2} \mathcal{P}_{1}^{(0)}+i \omega_{3} \tilde{r} \mathcal{V}_{0}^{(0) \prime}=0
$$

Here we have two cases.

The case a) $\mu=\nu=\omega$. The function $\mathcal{V}_{0}^{(0)}(t)$ is determined by (5.31). The equation has the particular solution in the form

$$
\mathcal{P}_{1}^{(0)}(t)=\left(M_{1}^{a} t^{2}+M_{2}^{a} t+M_{3}^{a}\right) \cos (\nu t)+\left(M_{4}^{a} t^{2}+M_{5}^{a} t+M_{6}^{a}\right) \sin (\nu t),
$$

where $M_{1}^{a}, M_{2}^{a}, M_{3}^{a}, M_{4}^{a}, M_{5}^{a}, M_{6}^{a}$ are some constants. We find these constants by substituting the particular solution into our equation:

$$
\begin{gathered}
M_{1}^{a}=0, \\
M_{2}^{a}=-\frac{i \omega_{3} \tilde{r} \hat{v}_{30}(\boldsymbol{\omega})}{2}+\frac{i \omega_{3} \tilde{r} \mathcal{A}_{3} \hat{\rho}_{e 0}}{4 \nu}-\frac{i \omega_{3} \tilde{r} \mathcal{A}_{3} \hat{\rho}_{e 0} \nu}{8 \nu^{2}}, \\
M_{3}^{a}=0 \\
M_{4}^{a}=-\frac{i \omega_{3} \tilde{r} \mathcal{A}_{3} \hat{\rho}_{e 0}}{8}, \\
M_{5}^{a}=-\frac{i \omega_{3} \tilde{r} \hat{v}_{31}(\boldsymbol{\omega})}{2 \nu}, \\
M_{6}^{a}=0 .
\end{gathered}
$$

That is, the general solution of the equation has the form

$$
\begin{aligned}
\mathcal{P}_{1}^{(0)}(t)= & \left(\left(-\frac{i \omega_{3} \tilde{r} \hat{v}_{30}(\boldsymbol{\omega})}{2}+\frac{i \omega_{3} \tilde{r} \mathcal{A}_{3} \hat{\rho}_{e 0}}{4 \nu}-\frac{i \omega_{3} \tilde{r} \mathcal{A}_{3} \hat{\rho}_{e 0} \nu}{8 \nu^{2}}\right) t+C_{5}\right) \cos (\nu t) \\
& +\left(-\frac{i \omega_{3} \tilde{r} \mathcal{A}_{3} \hat{\rho}_{e 0}}{8} t^{2}-\frac{i \omega_{3} \tilde{r} \hat{v}_{31}(\boldsymbol{\omega})}{2 \nu} t+C_{6}\right) \sin (\nu t) .
\end{aligned}
$$

The constants $C_{5}, C_{6}$ could be found by substituting this solution into the initial data of problem (5.28), but we do not need an exact form for these constants because we are interested only in terms which grow infinitely in time.

The case b) $\mu \neq \nu=\omega$. In this case the function $\mathcal{V}_{0}^{(0)}(t)$ is still determined by (5.31). The equation has the particular solution

$$
\mathcal{P}_{1}^{(0)}(t)=\left(M_{1}^{b} t+M_{2}^{b}\right) \cos (\nu t)+\left(M_{3}^{b} t+M_{4}^{b}\right) \sin (\nu t) .
$$


Substituting it into the equation, we find the constants $M_{1}^{b}, M_{2}^{b}, M_{3}^{b}, M_{4}^{b}$ :

$$
\begin{gathered}
M_{1}^{b}=-\frac{i \omega_{3} \tilde{r} \mathcal{A}_{3} \hat{\rho}_{e 0} \nu}{2\left(\mu^{2}-\nu^{2}\right)}, \\
M_{2}^{b}=-\frac{i \omega_{3} \tilde{r} \hat{v}_{31}(\boldsymbol{\omega})}{\mu^{2}-\nu^{2}}, \\
M_{3}^{b}=0, \\
M_{4}^{b}=\frac{i \omega_{3} \tilde{r} \hat{v}_{30}(\boldsymbol{\omega}) \nu}{\mu^{2}-\nu^{2}}-\frac{i \omega_{3} \tilde{r} \mathcal{A}_{3} \hat{\rho}_{e 0} \nu}{2\left(\mu^{2}-\nu^{2}\right)}-\frac{i \omega_{3} \tilde{r} \mathcal{A}_{3} \hat{\rho}_{e 0} \nu^{2}}{\left(\mu^{2}-\nu^{2}\right)^{2}} .
\end{gathered}
$$

That is, the general solution of the equation has the form

$$
\begin{aligned}
\mathcal{P}_{1}^{(0)}(t) & =C_{7} \cos (\mu t)+C_{8} \sin (\mu t)-\left(\frac{i \omega_{3} \tilde{r} \mathcal{A}_{3} \hat{\rho}_{e 0} \nu}{2\left(\mu^{2}-\nu^{2}\right)} t+\frac{i \omega_{3} \tilde{r} \hat{v}_{31}(\boldsymbol{\omega})}{\mu^{2}-\nu^{2}}\right) \cos (\nu t) \\
& +\left(\frac{i \omega_{3} \tilde{r} \hat{v}_{30}(\boldsymbol{\omega}) \nu}{\mu^{2}-\nu^{2}}-\frac{i \omega_{3} \tilde{r} \mathcal{A}_{3} \hat{\rho}_{e 0} \nu}{2\left(\mu^{2}-\nu^{2}\right)}-\frac{i \omega_{3} \tilde{r} \mathcal{A}_{3} \hat{\rho}_{e 0} \nu^{2}}{\left(\mu^{2}-\nu^{2}\right)^{2}}\right) \sin (\nu t) .
\end{aligned}
$$

As in the previous case we do not need an exact form of the constants $C_{7}$ and $C_{8}$.

The case c) $\mu=\omega \neq \nu$. The function $\mathcal{V}_{0}^{(0)}(t)$ is determined by (5.32). The equation has the particular solution

$$
\mathcal{P}_{1}^{(0)}(t)=M_{1}^{c} \cos (\nu t)+M_{2}^{c} \sin (\nu t)+M_{3}^{c} t \cos (\mu t) .
$$

The constants $M_{1}^{c}, M_{2}^{c}, M_{2}^{c}$ have the form

$$
\begin{gathered}
M_{1}^{c}=-\frac{i \omega_{3} \tilde{r} \hat{v}_{31}(\boldsymbol{\omega})}{\mu^{2}-\nu^{2}} \\
M_{2}^{c}=i \omega_{3} \tilde{r} \nu \frac{\hat{v}_{30}(\boldsymbol{\omega})\left(\mu^{2}-\nu^{2}\right)+\mathcal{A}_{3} \hat{\rho}_{e 0} \mu}{\left(\mu^{2}-\nu^{2}\right)^{2}} \\
M_{3}^{c}=\frac{i \omega_{3} \tilde{r} \mathcal{A}_{3} \hat{\rho}_{e 0} \mu}{2\left(\mu^{2}-\nu^{2}\right)} .
\end{gathered}
$$

Hence, the general solution of the equation is

$$
\begin{gathered}
\mathcal{P}_{1}^{(0)}(t)=\left(\frac{i \omega_{3} \tilde{r} \mathcal{A}_{3} \hat{\rho}_{e 0} \mu}{2\left(\mu^{2}-\nu^{2}\right)} t+C_{9}\right) \cos (\mu t)+C_{10} \sin (\mu t)-\frac{i \omega_{3} \tilde{r} \hat{v}_{31}(\boldsymbol{\omega})}{\mu^{2}-\nu^{2}} \cos (\nu t) \\
+i \omega_{3} \tilde{r} \nu \frac{\hat{v}_{30}(\boldsymbol{\omega})\left(\mu^{2}-\nu^{2}\right)+\mathcal{A}_{3} \hat{\rho}_{e 0} \mu}{\left(\mu^{2}-\nu^{2}\right)^{2}} \sin (\nu t) .
\end{gathered}
$$

Consider now case $\mathrm{d}$ ) when $\mu, \nu$, and $\omega$ are not equal to each other. The function $\mathcal{V}_{0}^{(0)}(t)$ is determined by (5.32), and the equation has the particular solution

$$
\mathcal{P}_{1}^{(0)}(t)=M_{1}^{d} \cos (\nu t)+M_{2}^{d} \sin (\nu t)+M_{3}^{d} \sin (\omega t) .
$$

The constants $M_{1}^{d}, M_{2}^{d}, M_{2}^{d}$ have the form

$$
\begin{gathered}
M_{1}^{d}=-\frac{i \omega_{3} \tilde{r} \hat{v}_{31}(\boldsymbol{\omega})}{\mu^{2}-\nu^{2}} \\
M_{2}^{d}=i \omega_{3} \tilde{r} \nu \frac{\hat{v}_{30}(\boldsymbol{\omega})\left(\omega^{2}-\nu^{2}\right)+\mathcal{A}_{3} \hat{\rho}_{e 0} \omega}{\left(\mu^{2}-\nu^{2}\right)\left(\omega^{2}-\nu^{2}\right)} \\
M_{3}^{d}=\frac{i \omega_{3} \tilde{r} \mathcal{A}_{3} \hat{\rho}_{e 0} \omega^{2}}{\left(\nu^{2}-\omega^{2}\right)\left(\mu^{2}-\omega^{2}\right)} .
\end{gathered}
$$


Then, the general solution is

$$
\begin{gathered}
\mathcal{P}_{1}^{(0)}(t)=C_{11} \cos (\mu t)+C_{12} \sin (\mu t)-\frac{i \omega_{3} \tilde{r} \hat{v}_{31}(\boldsymbol{\omega})}{\mu^{2}-\nu^{2}} \cos (\nu t) \\
+i \omega_{3} \tilde{r} \nu \frac{\hat{v}_{30}(\boldsymbol{\omega})\left(\omega^{2}-\nu^{2}\right)+\mathcal{A}_{3} \hat{\rho}_{e 0} \omega}{\left(\mu^{2}-\nu^{2}\right)\left(\omega^{2}-\nu^{2}\right)} \sin (\nu t)+\frac{i \omega_{3} \tilde{r} \mathcal{A}_{3} \hat{\rho}_{e 0} \omega^{2}}{\left(\nu^{2}-\omega^{2}\right)\left(\mu^{2}-\omega^{2}\right)} \sin (\omega t) .
\end{gathered}
$$

Let us now consider problem (5.26), (5.27). We will study the solution of this problem by the averaging method. We introduce the new variables

$$
\begin{aligned}
& Q=\mathcal{P}^{(1) \prime}-i \mu \mathcal{P}^{(1)}, \\
& V=\mathcal{V}^{(1) \prime}-i \nu \mathcal{V}^{(1)} .
\end{aligned}
$$

Then, problem (5.26), (5.27) has the form

$$
\begin{gathered}
\boldsymbol{X}^{\prime}=\tilde{A} \boldsymbol{X}+\hat{\Lambda} \boldsymbol{F}, \\
\left.\boldsymbol{X}\right|_{t=0}=0,
\end{gathered}
$$

where

$$
\begin{gathered}
\boldsymbol{X}=\left(\begin{array}{c}
\mathcal{P}^{(1)} \\
Q \\
\mathcal{V}^{(1)} \\
V
\end{array}\right), \\
\tilde{A}=\left(\begin{array}{cccc}
i \mu & 1 & 0 & 0 \\
0 & -i \mu & 0 & 0 \\
0 & 0 & i \nu & 1 \\
0 & 0 & 0 & -i \nu
\end{array}\right), \\
\boldsymbol{F}=\left(\begin{array}{c}
\hat{f}-i \omega_{3} \tilde{r}\left(V+i \nu \mathcal{V}^{(1)}\right) \\
0 \\
\hat{g}-i \omega_{3} \tilde{r}\left(Q+i \mu \mathcal{P}^{(1)}\right)
\end{array}\right) .
\end{gathered}
$$

Let us denote

$$
\boldsymbol{Y}=e^{-\tilde{A} t} \boldsymbol{X}
$$

Then $\boldsymbol{Y}$ satisfies the following Cauchy problem:

$$
\begin{gathered}
\boldsymbol{Y}^{\prime}=\hat{\Lambda} e^{-\tilde{A} t} \boldsymbol{F}, \\
\left.\boldsymbol{Y}\right|_{t=0}=0 .
\end{gathered}
$$

Let

$$
\mathcal{F}(t, \boldsymbol{\omega}, \hat{\Lambda}, \omega, \boldsymbol{Y})=e^{-\tilde{A} t} \boldsymbol{F} .
$$

We denote

$$
\mathcal{F}_{0}(t, \omega, \omega, \boldsymbol{Y})=\lim _{t \rightarrow \infty} \frac{1}{t} \int_{0}^{t} \mathcal{F}(t, \omega, 0, \omega, \boldsymbol{Y}) d t
$$


Consider the averaged problem

$$
\begin{aligned}
& \tilde{\boldsymbol{Y}}^{\prime}=\hat{\Lambda} \mathcal{F}_{0}, \\
& \left.\tilde{\boldsymbol{Y}}\right|_{t=0}=0 .
\end{aligned}
$$

It follows from Bogolyubov's theorem [11] that if $\boldsymbol{Y}(t)$ and $\tilde{\boldsymbol{Y}}(t)$ are the solutions of problems (5.31) and (5.39), respectively, then for any $\delta>0$ with a small enough $\hat{\Lambda}>0$ we have $|\boldsymbol{Y}(t)-\tilde{\boldsymbol{Y}}(t)|<\delta$ for $0 \leq t \leq \frac{K}{\hat{\Lambda}}$, where $K$ is a constant.

We now find solutions of problem (5.39). By applying the method of mathematical induction it is easy to prove that

$$
e^{-\tilde{A} t}=\left(\begin{array}{cccc}
e^{-i \mu t} & -\frac{\sin (\mu t)}{\mu} & 0 & 0 \\
0 & e^{i \mu t} & 0 & 0 \\
0 & 0 & e^{-i \nu t} & -\frac{\sin (\nu t)}{\nu} \\
0 & 0 & 0 & e^{i \nu t}
\end{array}\right) .
$$

We can also check that $\mathcal{F}_{0}$ is not identically zero only for $\omega=\mu$. In this case,

$$
\mathcal{F}_{0}=\left(\begin{array}{c}
-\frac{5}{8 \mu} R_{1} \hat{\rho}_{e 0} i \omega_{3} \\
-\frac{5}{8} R_{1} \hat{\rho}_{e 0} \omega_{3} \\
0 \\
0
\end{array}\right)
$$

Thus,

$$
\tilde{\boldsymbol{Y}}(t)=\hat{\Lambda}\left(\begin{array}{c}
-\frac{5}{8 \mu} R_{1} \hat{\rho}_{e 0} i \omega_{3} \\
-\frac{5}{8} R_{1} \hat{\rho}_{e 0} \omega_{3} \\
0 \\
0
\end{array}\right) t .
$$

From this formula we can easily get the following approximate solution of problem (5.26) (5.27):

$$
\begin{gathered}
\mathcal{P}^{(1)}=-i \frac{5 \hat{\Lambda}}{8 \mu} R_{1} \hat{\rho}_{e 0} \omega_{3} t \cos (\mu t), \\
\mathcal{V}^{(1)}=0 .
\end{gathered}
$$

Summarizing all the solutions, we obtain:

if $\mu=\omega, \mu=2 \omega$ and $\mu=\nu$, then $|\mathcal{P}|$ grows infinitely as $t \rightarrow \infty$;

if $\nu=\omega$, then $|\mathcal{V}|$ grows infinitely as $t \rightarrow \infty$.

6. Final remarks. We have proved the electrodynamical instability of layered structures at the presence of a small-amplitude alternating current, i.e., the linear instability (by Lyapunov) of this flow regime. This leads to the practical destruction of the basic solution. Thus, we can hope that the methods of electrometry could be effective for the destruction of layered structures and the restoration of the collector's permeability.

7. Acknowledgements. The authors are indebted to Dr. Yu. L. Trakhinin for help in the preparation of the manuscript of this paper. 


\section{REFERENCES}

[1] Dorovsky V.N., Dorovsky S.V. A hydrodynamic model of water-oil layered systems containing gas. Math. Comput. Modelling, 2002, v.35, pp.751-757.

[2] Dorovsky V.N., Belonosov V.S., Belonosov A.S. Numerical investigation of parametric resonance in water-oil structures containing gas. Math. Comput. Modelling, 2002, v.36, pp.203-209. MR.1925071

[3] Gogosov V.V, Polansky V.A. Electrohydrodynamics: problem and applications, fundamental equations, discontinuous solutions. Fluid mechanics (Resume of science and technics), 1976, 10, pp.5-85 (Russian).

[4] Blokhin A.M., Dorovsky V.N. Problems of the mathematical simulation in theory of the multivelocity continuum. RAS, Sib. dep, United Institute of geology, geophysics and mineralogy, Institute of Mathematics, Novosibirsk, 1994 (Russian).

[5] Kats E.N., Lebedev V.V. Liquid crystal dynamics. Moscow., Nauka, 1988 (Russian).

[6] Landau L.D., Lifshits E.M. Theoretical physics. v. VII. Theory of elasticity. Moscow, Nauka, 1987 (Russian). MR912888 (88m:00005)

[7] Landau L.D., Lifshits E.M. Theoretical physics. v. VI. Hydrodynamics. Moscow, Nauka, 1986 (Russian). MR850480 (87j:76001)

[8] Blokhin A.M., Dorovsky S.V. Shock waves stability in layered structures. Comp. and Math. with Appl., 2004, 47, pp.427-440. MR2048194 (2005j:35148)

[9] Blokhin A.M., Dorovsky S.V., Ovechkin E.V. Stability of shock waves in layered structures. Part 2. Comp. and Math. with Appl., 2004, 47, pp.1379-1387. MR.2070991(2005e:35236)

[10] Sedov L.N. Mechanics of continua, v.1. Moscow, Nauka, 1970 (Russian).

[11] Bogolyubov N.N., Mitropolskiy Yu.A. Asymptotic methods in the theory of the nonlinear oscillations. Moscow, Nauka, 1974 (Russian). MR0374550 (51:10750) 